\title{
CHANG-ES V: NUCLEAR OUTFLOW IN A VIRGO CLUSTER SPIRAL AFTER A TIDAL DISRUPTION EVENT
}

\author{
Judith A. Irwin ${ }^{1}$, Richard N. Henriksen ${ }^{1}$, Marita Krause ${ }^{2}$, Q. Daniel Wang ${ }^{3}$, Theresa Wiegert ${ }^{1}$, Eric J. Murphy ${ }^{4}$, \\ George Heald ${ }^{5}$, and Eric Perlman ${ }^{6}$ \\ ${ }^{1}$ Department of Physics, Engineering Physics, \& Astronomy, Queen's University, Kingston, Ontario, K7L 3N6, Canada; \\ irwin@astro.queensu.ca, henriksn@astro.queensu.ca, twiegert@astro.queensu.ca \\ ${ }^{2}$ Max-Planck-Institut für Radioastronomie, Auf dem Hügel 69, D-53121, Bonn, Germany; mkrause@mpifr-bonn.mpg.de \\ ${ }^{3}$ Department of Astronomy, University of Massachusetts, 710 North Pleasant Street, Amherst, MA 01003, USA; wqd@astro.umass.edu \\ ${ }^{4}$ US Planck Data Center, The California Institute of Technology, MC 220-6, Pasadena, CA 91125, USA; emurphy@ipac.caltech.edu \\ ${ }^{5}$ Netherlands Institute for Radio Astronomy (ASTRON), Postbus 2, 7990 AA, Dwingeloo, The Netherlands; heald@ astron.nl \\ ${ }^{6}$ Physics and Space Sciences Department, Florida Institute of Technology, 150 West University Boulevard, Melbourne, FL 32901, USA; eperlman@ fit.edu \\ Received 2015 February 8; accepted 2015 July 2; published 2015 August 21
}

\begin{abstract}
We have observed the Virgo Cluster spiral galaxy, NGC 4845, at 1.6 and $6 \mathrm{GHz}$ using the Karl G. Jansky Very Large Array, as part of the Continuum Halos in Nearby Galaxies-an EVLA Survey (CHANG-ES). The source consists of a bright unresolved core with a surrounding weak central disk ( $1.8 \mathrm{kpc}$ diameter). The core is variable over the 6 month timescale of the CHANG-ES data and has increased by a factor of $\approx 6$ since 1995 . The wide bandwidths of CHANG-ES have allowed us to determine the spectral evolution of this core, which peaks between 1.6 and $6 \mathrm{GHz}$ (it is a Gigahertz-peaked spectrum source). We show that the spectral turnover is dominated by synchrotron self-absorption and that the spectral evolution can be explained by adiabatic expansion (outflow), likely in the form of a jet or cone. The CHANG-ES observations serendipitously overlap in time with the hard X-ray light curve obtained by Nikolajuk \& Walter (2013), which they interpret as due to a tidal disruption event (TDE) of a super-Jupiter mass object around a $10^{5} M_{\odot}$ black hole. We outline a standard jet model, provide an explanation for the observed circular polarization, and quantitatively suggest a link between the peak radio and peak X-ray emission via inverse Compton upscattering of the photons emitted by the relativistic electrons. We predict that it should be possible to resolve a young radio jet via VLBI as a result of this nearby TDE.
\end{abstract}

Key words: galaxies: active - galaxies: individual (N4845) - galaxies: nuclei

\section{INTRODUCTION}

The discovery of a hard X-ray source at the center of the galaxy, NGC 4845, by INTEGRAL (IGR J12580+0134) has been interpreted as the tidal disruption of a super-Jupiter by a massive black hole (Nikolajuk \& Walter 2013). As part of the Continuum Halos in Nearby Galaxies-an EVLA Survey (CHANG-ES), ${ }^{7}$ we have detected a variable radio source (a compact core) in NGC 4845 (Table 1), showing unambiguously that this galaxy harbors an active galactic nucleus (AGN). The peak of the X-ray light curve occurred on 2011 January 22. Our radio observations were carried out approximately one year later (Table 2) and overlap with the time period of their light curve (their Figure 8).

The radio spectrum peaks at Gigahertz frequencies, like the well-known Gigahertz-peaked spectrum (GPS) sources seen at high redshift (e.g., O'Dea 1998; Tornikoski et al. 2001). Characteristic of such sources are their small radio sizes, which are generally thought to be because they are young radio sources, or confined by a surrounding medium, or both (Fanti 2009; Mingaliev et al. 2013).

NGC 4845 is located in the "Virgo Southern Extension" (Tully 1982; Karachentsev \& Nasonova 2013; $D=17 \mathrm{Mpc}$, Table 1), providing a unique opportunity to study the radio/Xray connection for this nearby AGN. A similar relevant case (Zauderer et al. 2011) is the radio/X-ray analysis discussing the "birth of a relativistic outflow" in a $\gamma$-ray transient at a redshift of $z=0.354$. NGC 4845 is 100 times closer and, with future monitoring, may provide an unprecedented opportunity

\footnotetext{
7 The Expanded Very Large Array is now known as the Karl G. Jansky Very Large Array.
}

to follow the development of an outflow in what could be a present day lower-luminosity version of a newly forming quasar.

In this paper, we present our observations and develop a simple cone/jet model that is consistent with the data, including the variability of the radio flux density and spectral index, the behavior of the radio spectrum, the observed circular polarization, and the absence of significant linear polarization. We also suggest a possible link between the peak X-ray and peak radio emission in the context of our model.

Table 2 provides imaging parameters from our five data sets as well as a summary of previous radio observations from the literature, clearly illustrating the variability of the source. It is not surprising that NGC 4845 has been variously listed as having an H II/LINER type nucleus, provided by the NASA extragalactic database (NED) based on optical spectra of Ho et al. 1995), a pure $\mathrm{H}$ II region type nucleus (Filho et al. 2000), and a Seyfert 2 nucleus (Véron-Cetty \& Véron 2010), given that the relative importance of the nucleus/disk changes with time. In that respect, NGC 4845 appears to be a closer, fainter version of the recently identified "changing-look quasar" in which the nuclear activity has changed from Type 1 to a Type 1.9 AGN over a 10 year timespan (LaMassa et al. 2015).

Section 2 presents the observations and data reductions, Section 3.1 outlines the total intensity results, in-band spectral indices, and variability of the source with fitted spectra, and Section 3.2 gives the polarization results. Section 4 discusses the core. We provide a detailed analysis of the core spectra and their variability and also present several appendices (Appendices $\mathrm{A}, \mathrm{B}$, and $\mathrm{E}$ ) that not only form a description and are consistent with the observations, but also summarize more 
Table 1

Galaxy Parameters

\begin{tabular}{lc}
\hline \hline Parameter & Value \\
\hline Distance $(\mathrm{Mpc})^{\mathrm{a}}$ & 17 \\
${\text { Inclination }(\mathrm{deg})^{\mathrm{b}}}$ & 81 \\
$V_{\odot}\left(\mathrm{km} \mathrm{s}^{-1}\right)^{\mathrm{c}}$ & 1098 \\
$\Delta V\left(\mathrm{~km} \mathrm{~s}^{-1}\right)^{\mathrm{d}}$ & $374 \pm 0.4$ \\
$\mathrm{HI} \mathrm{mass}\left(M_{\odot}\right)^{\mathrm{e}}$ & $(2.1 \pm 0.4) \times 10^{8}$ \\
Total mass $\left(M_{\odot}\right)^{\mathrm{f}}$ & $9.9 \times 10^{10}$ \\
$I_{\mathrm{CO}}\left(\mathrm{K} \mathrm{km} \mathrm{s}^{-1}\right)^{\mathrm{g}}$ & 6.07 \\
\hline
\end{tabular}

Notes.

a Solanes et al. (2002).

b Irwin et al. (2012a).

${ }^{c}$ Heliocentric velocity (Springob et al. 2005). Note that this (and subsequent HI related parameters) are adjustments from Irwin et al. (2012a).

${ }^{d}$ From the double-horned profile of Springob et al. (2005), corrected for redshift stretch, instrumental effects, and smoothing.

e Springob et al. (2005), as corrected for pointing offsets, source extent, and HI self-absorption.

${ }^{\mathrm{f}}$ Using $\Delta V$ and the inclination, above, assuming a spherical mass distribution, and an $\mathrm{HI}$ radius equivalent to the optical blue diameter of $4.8 \mathrm{arcmin}$ (the $\mathrm{HI}$ angular extent is unknown).

${ }^{\mathrm{g}} \mathrm{CO}$ integrated intensity using Five Colleges Radio Astronomy Observatory data (Komugi et al. 2008).

general concepts that may be useful for future observations of NGC 4845 and other such sources, especially in an era in which the sensitivity, fidelity, and resolution of radio data are rapidly improving. Appendix F links the X-ray and radio data. The conclusions are presented in Section 5.

\section{OBSERVATIONS AND DATA REDUCTIONS}

As part of the CHANG-ES program, five observations were carried out (B, C, and D configurations at L-band, and C and D configurations at $\mathrm{C}$-band) in all polarization products over approximately a six month period (Table 2). Hereafter we will refer to each observation according to "configuration/band" (i.e., B/L, C/L, D/L, C/C, and D/C). Data reduction and imaging details are described in Irwin et al. (2013) and Wiegert et al. (2015). Here we provide a brief outline, as well as details specific to NGC 4845. The Common Astronomy Software Applications (CASA) package (McMullin et al. 2007) was used throughout. $^{8}$

For each observation, the galaxy was observed within a scheduling block (SB) that included other galaxies so the uv coverage could be maximized for each. For $\mathrm{C} / \mathrm{C}$, however, the galaxy was observed in two separate SBs on different dates, which were calibrated separately and then combined for imaging.

The calibrators were 3C 286 (the "primary" calibrator), which was also used for the bandpass and polarization angle calibration, and J1246-0730 (or J1224+0330 for C/C), (the "secondary" calibrator), which is near the source on the sky. To determine the leakage terms between the $\mathrm{R}$ and $\mathrm{L}$ circularly polarized feeds for polarization imaging, we used the unpolarized source, $\mathrm{J} 1407+2827=\mathrm{OQ} 208$, except for

\footnotetext{
8 See also http://casa.nrao.edu.

9 The secondary calibrator was used in the event that OQ 208 carried some polarization at C-band. A test using both methods, however, showed negligible differences between the results.
}

C-band, for which we used the secondary calibrators, which spanned a parallactic angle $>60^{\circ}$ over the observations. ${ }^{9}$

At L-band, the frequency coverage was (in $\mathrm{GHz}$ ) $1.247 \rightarrow 1.503$ and $1.647 \rightarrow 1.903(500 \mathrm{MHz}$ total $)$; at C-band it was $4.979 \rightarrow 7.021(2 \mathrm{GHz})$. The frequency gap at L-band was set to avoid very strong persistent interference in that frequency range.

Flagging was carried out iteratively (i.e., after each round of calibration, the data were re-inspected, more flagging was carried out if needed, the calibration tables were remade, the data were recalibrated, and so on). Any flagging that was carried out for the total intensity data (RR and LL circular polarizations) was also automatically applied to the cross-hands (RL and LR). In addition, for the polarization calibration $(Q$ and $U$ maps, see below), RL and LR data were inspected, and flagging/calibration iterations were again carried out prior to polarization imaging.

Wide-field imaging (Cornwell et al. 2008) using the multiscale, multi-frequency-synthesis algorithm (Cornwell 2008; Rau \& Cornwell 2011) was also carried out, with simultaneous fitting of a simple power law $\left(S_{\nu} \propto \nu^{\alpha}\right)$ across the band. However, because NGC 4845 is point-like (Section 3.1), the use of many scales was not warranted. After a variety of trials, we found that a classic clean gave the best results for highly unresolved cases $(\mathrm{D} / \mathrm{L}, \mathrm{C} / \mathrm{L}, \mathrm{D} / \mathrm{C})$, with the addition of modest scales for slightly resolved cases (B/L: 0, 5, 10, and 20 arcsec; C/C: 0 and 5 arcsec).

For all maps, Briggs robust $=0$ uv weighting was used (Briggs 1995), as implemented in CASA. A variety of selfcalibration steps were then employed, either phase-only $(\mathrm{P})$ or amplitude and phase together (A\&P), depending on the specific data set and what was required to improve the dynamic range (DR) of the map. Care was taken to ensure that, with successive iterations, the self-calibration did not go so deep as to start including spurious values, such as negatives or residual sidelobes, and that the peak specific intensity did not decline. The primary beam (PB) correction was then applied. Table 2 summarizes the imaging parameters.

For each observation, imaging of Stokes $Q, U$, and $V$ was also carried out using the same self-calibration tables and the same input parameters as for total intensity (except $\mathrm{B} / \mathrm{L}$, for which the scales were reduced to 0,5 , and $20 \operatorname{arcsec}$ ). Maps of linear polarization, $P=\sqrt{Q^{2}+U^{2}}$, were then made and corrected for the bias introduced by the fact that $\mathrm{P}$ images do not obey Gaussian statistics (e.g., Simmons \& Stewart 1985; Vaillancourt 2006) as well as maps of polarization angle. $P$ and $V$ maps were then corrected for the PB.

In the case of circular polarization, we also remade the maps without fitting a spectrum across the band, essentially averaging all spectral channels together without attempting to fit a spectral index. However, those maps were significantly worse than when a spectral fit was carried out. For example, at $\mathrm{C} / \mathrm{L}$, including a spectral fit increased the peak specific intensity by $5 \%$ and decreased the rms noise by $32 \%$. We therefore retained the $V$ maps that included a spectral fit.

For each observation, in-band spectral index, $\alpha$ maps, and $\alpha$ error maps were also made (Rau \& Cornwell 2011), as implemented in the CASA clean algorithm. Following this, the post-imaging prescription of Wiegert et al. (2015) was followed, which ensures that the images are corrected for the spectral index that is imposed by the PB (e.g., see also Irwin et al. 2012b, 2013) and smooths over artifacts. However we 
Table 2

Image Parameters

\begin{tabular}{|c|c|c|c|c|c|c|c|c|}
\hline Observation & Date $^{a}$ & $\begin{array}{c}\nu_{0}^{\mathrm{b}} \\
(\mathrm{GHz})\end{array}$ & $\begin{array}{c}\text { Beam Parameters }{ }^{c} \\
\left({ }^{\prime \prime}, ", \text { deg. }\right)\end{array}$ & $\begin{array}{l}\text { Pixel Size } \\
\left({ }^{\prime \prime}\right)\end{array}$ & SC Iterations ${ }^{\mathrm{d}}$ & $I$ & $\begin{array}{c}\mathrm{rms}^{\mathrm{e}} \\
Q, U \\
\left(\mu \mathrm{Jy}^{\left.- \text {beam }^{-1}\right)}\right.\end{array}$ & $V$ \\
\hline D array L-band & 2011 Dec 30 & 1.57470 & $38.58,34.27,-5.22$ & 5.0 & $2 \mathrm{~A} \& \mathrm{P}$ & 40 & 27 & 28 \\
\hline $\mathrm{C}$ array L-band & 2012 Mar 30 & 1.57484 & $12.18,11.10,-41.76$ & 1.0 & $2 \mathrm{~A} \& \mathrm{P}$ & 45 & 19 & 26 \\
\hline B array L-band & 2012 Jun 11 & 1.57499 & $3.51,3.33,22.69$ & 0.5 & $2 \mathrm{~A} \& \mathrm{P}$ & 18 & 15 & 15 \\
\hline $\mathrm{C}$ array $\mathrm{C}$-band ${ }^{\mathrm{f}}$ & 2012 Feb 23 and 25 & 5.99854 & $3.05,2.75,-11.71$ & 0.5 & $1 \mathrm{P}+5 \mathrm{~A} \& \mathrm{P}$ & 3.9 & 3.2 & 3.3 \\
\hline \multicolumn{9}{|l|}{ Previous values } \\
\hline D array L-band (NVSS) $)^{g}$ & 1995 Feb 27 & 1.4 & $45,45,0$ & 15.0 & $\cdots$ & 450 & $\cdots$ & $\cdots$ \\
\hline \multirow[t]{2}{*}{ Arecibo $^{\mathrm{h}}$} & 1975 Aug $28 \rightarrow 1975$ Sep 11 & 2.380 & $\sim 162^{\mathrm{i}}$ & $\cdots$ & $\cdots$ & $\cdots$ & $\cdots$ & $\cdots$ \\
\hline & 1975 Dec $21 \rightarrow 1976$ Jan 03 & $\ldots$ & $\ldots$ & $\cdots$ & $\cdots$ & $\cdots$ & $\cdots$ & $\cdots$ \\
\hline
\end{tabular}

Notes. New CHANG-ES data are separated from prevously measured values (where available) by the double-line.

${ }^{\text {a }}$ Date (or range) that the observations were carried out (UTC, if time specified).

${ }^{\mathrm{b}}$ Central frequency of each image. Slight differences for the same band result from different amounts of flagging in different data sets. The bandwidths (GHz) were $1.247 \rightarrow 1.503$ and $1.647 \rightarrow 1.903$ at L- and C-bands, respectively

${ }^{c}$ Major and minor axis diameters and position angle of the synthesized beam.

${ }^{\mathrm{d}}$ Each application of a self-calibration iteration acted on the non-self-calibrated data, but using a model that improved with each iteration.

${ }^{\mathrm{e}} \mathrm{rms}$ map noise measured in a consistent fashion for all maps from random regions that do not appear to contain background sources. For $\mathrm{D} / \mathrm{L}$ and $\mathrm{C} / \mathrm{L}$ total intensity images only, the rms noise is higher by a factor of 1.5-2 close to the source itself because of residual sidelobes and the presence of many background sources.

${ }_{\mathrm{f}}$ These observations were split over two dates as specified.

g Downloaded data from the NRAO VLA Sky Survey (NVSS; Condon et al. 1998) website (http://www.cv.nrao.edu/nvss/).

${ }^{\text {h }}$ Dressel \& Condon (1978). The observations were carried out during one of the two date intervals specified.

${ }^{\mathrm{i}}$ Assuming a zenith angle of $10^{\circ}$.

${ }^{\mathrm{j}}$ Downloaded data from the Faint Images of the Radio Sky at Twenty-Centimeters (FIRST) survey (Becker et al. 1995) website (http://sundog.stsci.edu/index.html).

${ }^{\mathrm{k}}$ Filho et al. (2000), using beam parameters corresponding to their most reliable flux density. Calibration errors are quoted as a few percent.

${ }^{1}$ Harnett (1982).

departed from that prescription by being more conservative in our cutoff (i.e., spectral index maps for NGC 4845 were cut off wherever the total intensity fell below $10 \sigma$ instead of $5 \sigma$; the latter is the norm for CHANG-ES).

A weighted mean spectral index, $\bar{\alpha}$, and its associated error, $\overline{\Delta \alpha}$, were calculated over a region of interest, which was taken to be only the FWHM of a single beam. Essentially, this means we are adopting a measure of $\alpha$ in a single beam at the galaxy center, which is a region in which variations in the spectral index have been minimized. The values are calculated via:

$$
\begin{gathered}
\bar{\alpha}=\left\{\frac{\Sigma_{i}\left(\alpha_{i} / \Delta \alpha_{i}^{2}\right)}{\Sigma_{i}\left(1 / \Delta \alpha_{i}^{2}\right)}\right\} \\
\overline{\Delta \alpha}=\left\{\frac{N_{\mathrm{p} / \mathrm{b}}}{\Sigma_{i}\left(1 / \Delta \alpha_{i}^{2}\right)}\right\}^{1 / 2}
\end{gathered}
$$

where the summations are over the map pixels in the region of interest (i.e., the central beam). The factor, $N_{\mathrm{p} / \mathrm{b}}$, is the number of pixels per beam, which increases the error because the pixels are not independent. These weightings are adopted to ensure that pixels with the highest signal-to-noise ratio and lowest uncertainty are given the highest weight. Since the source is mainly unresolved (Section 3.1), the weighting is close to that of a Gaussian. The beam size is shown in lower left of Figures 3 and 4.

The results are given in Table 3. It is important to point out that the DR of each total intensity map is very high. The DRs are $5600 / 1(\mathrm{D} / \mathrm{L}), 5460 / 1(\mathrm{C} / \mathrm{L})$, and $11,600 / 1(\mathrm{~B} / \mathrm{L})$ at $\mathrm{L}$-band (but note that for $\mathrm{D} / \mathrm{L}$ and $\mathrm{C} / \mathrm{L}$, the rms is higher close to the source; see notes to Table 2). At C-band, the measured DR is higher still, namely 28,300/1 (D/C) and 88,900/1 $(\mathrm{C} / \mathrm{C})$, although other limitations at the VLA suggest practical limits of order 10,000/1 (S. Bhatnager 2012, private communication). As a result, the formal errors are quite small. However, tests have shown that the true error in the spectral index for a typical CHANG-ES galaxy is $\approx 20 \%$ higher (Wiegert et al. 2015). In addition, if we shift the measuring region up or down slightly from the center for our C/L and D/ $\mathrm{L}$ data sets, then $\bar{\alpha}$ will change because of their observed northsouth gradients (Figure 3). We ensured that all data sets are measured at the same position and in the same way, and in Section 3.1.5 we outline a number of checks indicating that possible variations in alpha do not affect the conclusions of this paper. 
Table 3

In-band Spectral Indices

\begin{tabular}{|c|c|c|c|c|}
\hline Observation & Date & $\bar{\alpha}$ & $\overline{\alpha_{\mathrm{AGN}}}$ & $\overline{\alpha_{V}}$ \\
\hline D array L-band & 2011 Dec $30\left(T_{1}\right)$ & $+0.95 \pm 0.01$ & $+1.11 \pm 0.02$ & $-2.2 \pm 0.1$ \\
\hline $\mathrm{C}$ array L-band & 2012 Mar 30 & $+0.97 \pm 0.01$ & $+1.11 \pm 0.02$ & $-2.5 \pm 0.2$ \\
\hline B array L-band & 2012 Jun 11 & $+0.81 \pm 0.17$ & $+0.81 \pm 0.17$ & $-3.4 \pm 0.1$ \\
\hline D array C-band & 2011 Dec 19 & $-0.453 \pm 0.003$ & $-0.448 \pm 0.006$ & $\cdots$ \\
\hline $\mathrm{C}$ array $\mathrm{C}$-band & 2012 Feb 23 and 25 & $-0.493 \pm 0.001$ & $-0.493 \pm 0.001$ & $\ldots$ \\
\hline
\end{tabular}

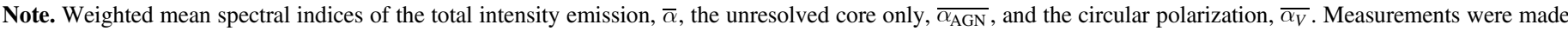

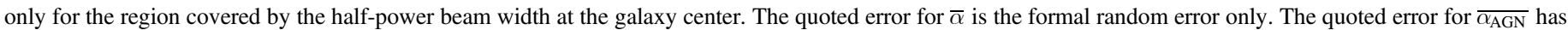

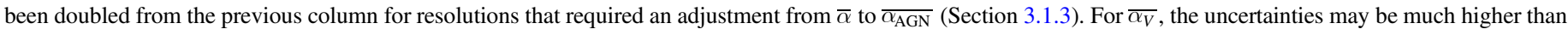
the formal error and thus all values of $\overline{\alpha_{V}}$ are taken to be roughly the same.

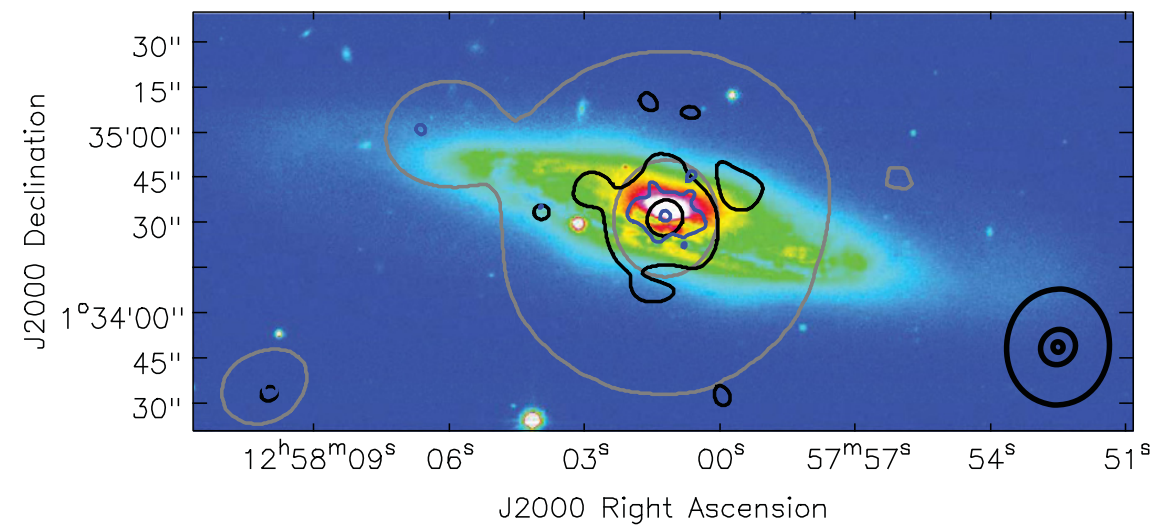

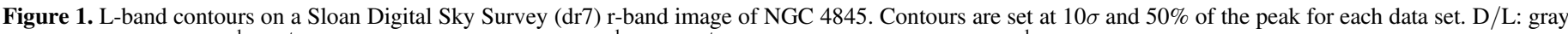
$\left(0.40,112.4 \mathrm{mJy}_{\text {beam }}{ }^{-1}\right), \mathrm{C} / \mathrm{L}$ : black $\left(0.45,122.8 \mathrm{mJy}\right.$ beam $\left.^{-1}\right)$, and B/L: blue $\left(0.18,104.7 \mathrm{mJy} \mathrm{beam}^{-1}\right)$. The beam sizes are shown at lower right.

Spectral index maps for the circularly polarized emission (not shown) were formed and measured in the same way.

\section{RESULTS}

\subsection{Total Intensity Emission}

In Figure 1, we show simple contours ( $10 \sigma$ and halfmaximum only) of the L-band emission for all three array configurations on an r-band image of NGC 4845 from the Sloan Digital Sky Survey (SDSS). ${ }^{10}$ It is clear that the radio emission is unresolved (cf. the beams at lower right) with the exception of a small disk around the nucleus showing up at the highest resolution $(\mathrm{B} / \mathrm{L})$. We will refer to this disk as the "central disk." In no case, however, do we see emission from an extended disk (Section 3.1.6) (i.e., over a size that might approach the optical extent of the galaxy).

Figure 2 shows C-band contours on a blow-up of the same optical emission. This image clearly shows asymmetric optical emission in the shape of a cone to the north of the nucleus. The galaxy is slightly inclined (Table 1) such that its southern edge is closest to us; consequently, a corresponding southern optical cone, if it exists, would be obscured by the disk.

Again, the $\mathrm{D} / \mathrm{C}$ (red contours) radio emission is unresolved but the $\mathrm{C} / \mathrm{C}$ emission (black contours) delineates the small central disk quite well. The disk subtends $21.7 \operatorname{arcsec}(1.8 \mathrm{kpc}$ in diameter) to the displayed $10 \sigma$ contour level at a position angle of $83^{\circ}$, which is in contrast to the larger-scale optical disk that shows some distortion and whose position angle varies

${ }_{10}$ http://www.sdss.org/

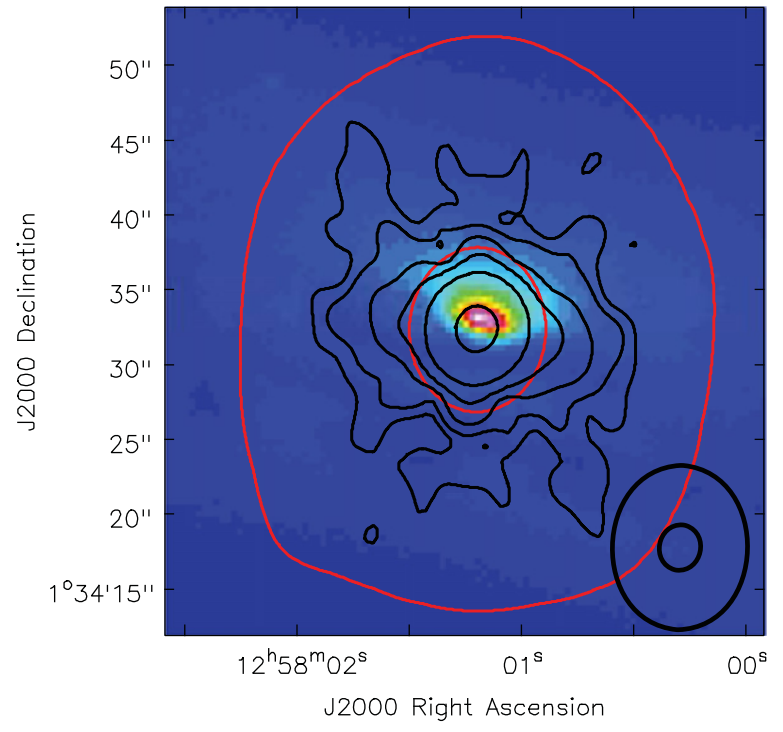

Figure 2. C-band contours on a blow-up of the optical emission as shown in Figure 2. Contours are red for $\mathrm{D} / \mathrm{C}$ at $0.15(10 \sigma)$ and $212.4 \mathrm{mJy}_{\text {beam }}{ }^{-1}$ (halfpeak value). Contours are black for $\mathrm{C} / \mathrm{C}$ at $0.039,0.10,0.30,1.00,6.00$, and $173.1 \mathrm{mJy}^{-1}$ beam $^{-1}$ (half-peak value). The beam sizes are shown at lower right.

from $74^{\circ}$ to $80^{\circ}$, depending on the isophotes to which it is measured. A similarly angled radio disk is visible at $8.4 \mathrm{GHz}$, whose size $(\approx 20 \operatorname{arcsec})$ agrees with our result (Filho et al. 2000).

The central disk shows some extensions to the north and south $(\mathrm{B} / \mathrm{L}$ and $\mathrm{C} / \mathrm{C}$, Figures 1 and 2$)$ that could be related to 
Table 4

Flux Densities

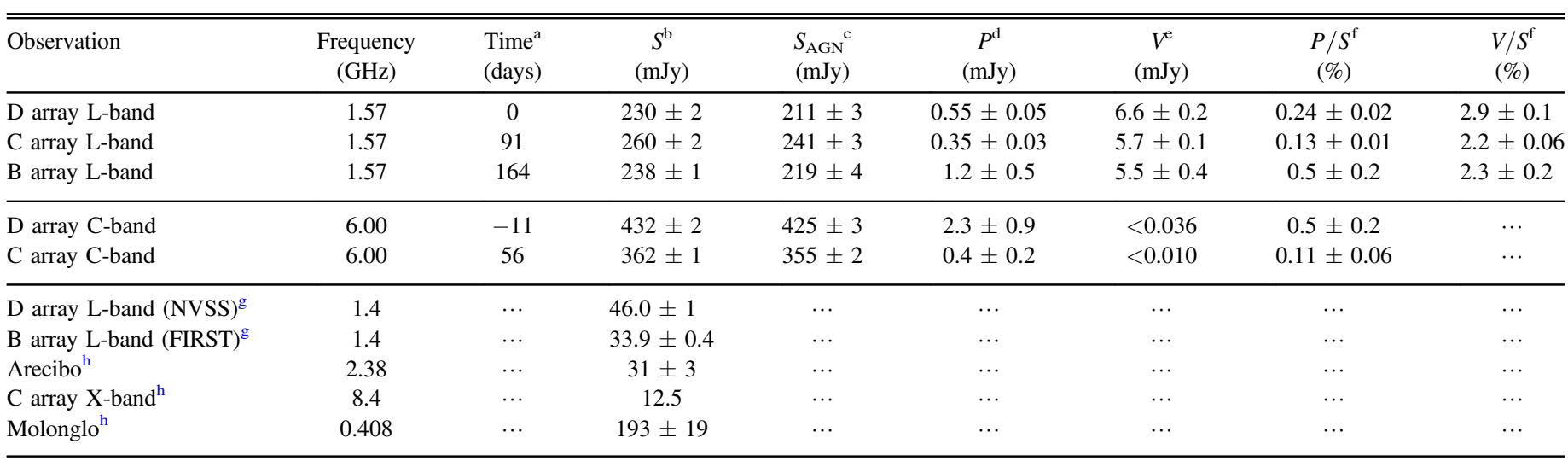

Notes. Flux densities for the data sets of Table 2. Frequencies have been rounded. Upper limits assume that the source is unresolved and that a detection would require a $3 \sigma$ signal, where $\sigma$ is the rms noise of the map (Table 2).

a Time in days since $T_{1}=2011$ December 30 .

${ }^{\mathrm{b}}$ Flux density of the total intensity emission. The quoted error does not include the $1 \%$ absolute flux density error of $3 \mathrm{C} 286$. See Section 3.1 .1 for details.

${ }^{\mathrm{c}}$ Flux density of the AGN after subtracting off the disk emission. The latter was determined by subtracting off Gaussian fits to the peak of the highest resolution data at each frequency (Section 3.1.1).

${ }^{\mathrm{d}}$ Linearly polarized flux density, $P=\sqrt{Q^{2}+U^{2}}$, where $P$ has been corrected for positive bias. $P$ has been measured in varying-sized regions and, where the emission was point-like ( $\mathrm{D} / \mathrm{L}$ and $\mathrm{C} / \mathrm{L})$, via Gaussian fits as well. The quoted error reflects the resulting variations.

${ }^{\mathrm{e}}$ Flux density of the circularly polarized emission. Errors are random only, i.e., as evaluated from the rms noise and residual sidelobes in the map itself. See Section 3.2.

${ }^{\mathrm{f}}$ Percentage polarization of either the linearly or circularly polarized emission. For $V / S$, the C-band results have been omitted because the $V$ values are non-detections. ${ }^{g}$ Flux densities from downloaded data sets (FIRST and NVSS) were measured in the same way as for our data. The NVSS value agrees with the result from Condon et al. (1998) and the FIRST value agrees with Becker et al. (2012).

${ }^{\mathrm{h}}$ See Table 2 for references.

outflow. Filho et al. (2000) similarly note a small northern extension at $8.4 \mathrm{GHz}$.

\subsubsection{Flux Densities}

Flux densities were measured from the PB corrected images and are given in Table 4 along with previous literature values. With the exception of the $\mathrm{D} / \mathrm{C}$ data set (see below), the quoted errors are dominated by variations in flux density that result from adjusting the box size around the source. It is abundantly clear that NGC 4845 is a variable radio source, as its L-band flux density, $S$, has increased approximately 6-fold since the NVSS survey results in 1995.

Because we will also argue that the flux density has varied over the several-month timescale of our own observations, it is important to consider possible uncertainties that may not have been taken into account from the map measurements. These include (a) missing flux due to missing spatial scales, (b) calibration errors, and (c) changes in flux density from selfcalibration.

For (a), the largest angular scale detected at L-band is 16 arcmin; at C-band it is 4 arcmin. The structures shown in Figures 1 and 2 are well within these limits, indicating that all flux has been detected in each configuration/frequency-band combination. Calibration errors (b), as measured during the transfer from the primary to the secondary calibrator, are less than the errors quoted in Table 4 except for $\mathrm{D} / \mathrm{C}$ for which they dominate and are quoted in the table. The uncertainties of Table 4 do not take into account uncertainties in the primary calibrator (3C 286) itself (Perley \& Butler 2013; of order 1\%), but this will not affect comparisons between data sets. Selfcalibration (c) can result in some modification of the flux density. The largest such change occurred for the $\mathrm{C} / \mathrm{L}$ data set, for which a $4 \mathrm{mJy}$ difference is observed between the non-selfcalibated data (256 mJy) and the self-calibrated data (260 mJy), an increase of $1.5 \%$. Only a decrease in flux density would be a concern and any decrease observed had a smaller relative error than this. Self-calibration, for all data sets, resulted in an increase in DR and a lower rms noise.

Because the emission was point-like, the flux densities were also measured by fitting a Gaussian to the emission; the result agreed with the tabulated values, except for the high resolution data $(\mathrm{B} / \mathrm{L}$ and $\mathrm{C} / \mathrm{C})$ for which some disk emission is also seen and therefore the Gaussian fitted flux densities slightly underestimate the total flux density $(92 \%$ and $98 \%$ for $\mathrm{B} / \mathrm{L}$ and $\mathrm{C} / \mathrm{C}$, respectively). This also allows us to separate the flux density of the non-varying disk from the AGN, thereby isolating the flux density of the variable AGN alone, $S_{\mathrm{AGN}}$ (Table 4). From the global flux densities of the disk alone at the two frequencies $(19 \pm 4 \mathrm{mJy}$ at L-band and $7 \pm 1 \mathrm{mJy}$ at C-band), we find that the spectral index for the disk is $\alpha_{\text {disk }}=-0.74$. In general, such a value is typical of galaxy disks.

\subsubsection{Core Size and Astrometry}

The Gaussian fits discussed in the previous section were also used to determine the size and astrometry of the central core (which we take to represent the AGN), using the highest resolution data $(\mathrm{C} / \mathrm{C}$ and $\mathrm{B} / \mathrm{L}$; Table 5$)$. The average central position of the source is at R.A. $=12^{\mathrm{h}} 58^{\mathrm{m}} 01^{\mathrm{s}} .1968 \pm 0.0007$ $\left( \pm 0^{\prime \prime} .01\right)$, decl. $=01^{\circ} 34^{\prime} 32^{\prime \prime} .42 \pm 0^{\prime \prime} .01$, where we adopt the higher of the uncertainties from the two data sets. ${ }^{11}$ This is the most precise position yet determined for the core of NGC 4845

$\overline{11}$ These are formal errors from the CASA imfit task. 
Table 5

Source Astrometry

\begin{tabular}{lccc}
\hline \hline Observation & $\begin{array}{c}\nu_{0}^{\mathrm{a}} \\
(\mathrm{GHz})\end{array}$ & $\begin{array}{c}\text { R.A., Decl. }(\mathrm{J} 2000)^{\mathrm{b}} \\
\left(\mathrm{h}: \mathrm{m}: \mathrm{s}, \mathrm{o}^{\prime}:^{\prime \prime}\right)\end{array}$ & $\begin{array}{c}\text { Source Size }^{\mathrm{c}} \\
\left(^{\prime \prime},{ }^{\prime \prime}, \text { deg. }\right)\end{array}$ \\
\hline B array L-band & 1.57499 & $12: 58: 01.1955 \pm 0.0007,+01: 34: 32.42 \pm 0.01$ & $0.67 \pm 0.03,0.15 \pm 0.09,87 \pm 170$ \\
C array C-band & 5.99854 & $12: 58: 01.1981 \pm 0.0001,+01: 34: 32.424 \pm 0.001$ & $0.40 \pm 0.03,0.36 \pm 0.03,97 \pm 175$ \\
\hline
\end{tabular}

Notes.

${ }^{\text {a }}$ Reference frequency for each image, as in Table 2 .

${ }^{\mathrm{b}}$ Positions from Gaussian fits. The quoted uncertainty is the larger of the formal errors or variations resulting from the adoption of three different boxes within which the fit was carried out.

${ }^{\mathrm{c}}$ Deconvolved from the beam, from Gaussian fits. The quoted uncertainty is as described in note $b$. The fit includes an offset to account for the fact that the AGN is within a disk.
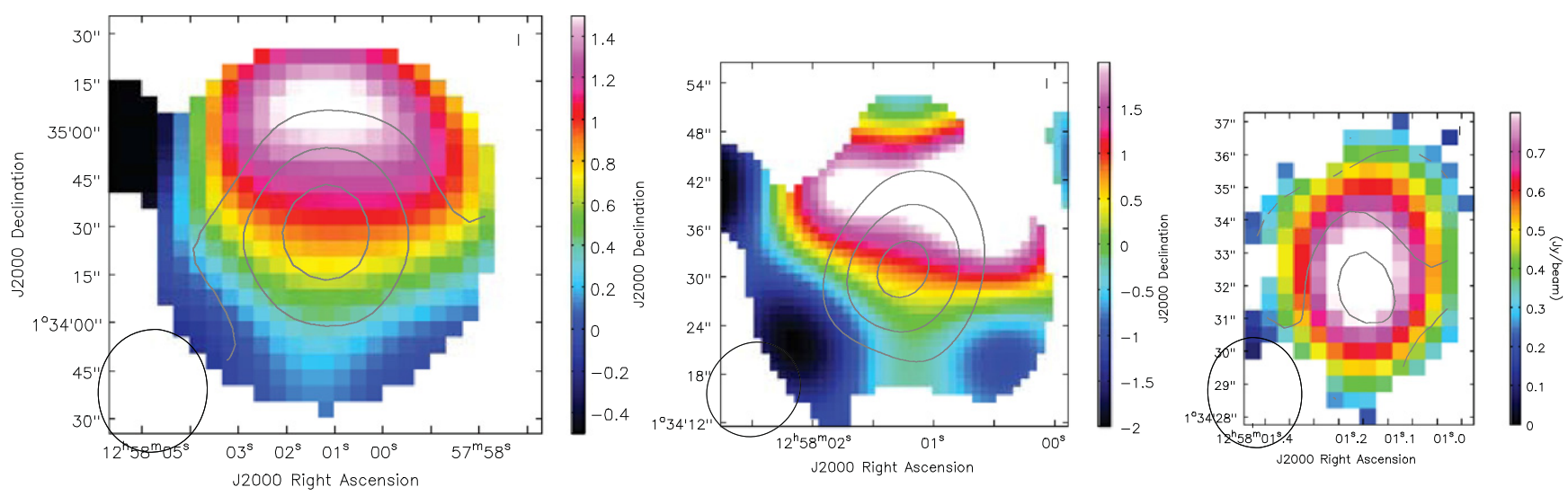

Figure 3. L-band spectral index maps formed as described in Section 2. Colors represent the spectral index with the color legend shown at right. Gray solid contours represent values from the corresponding error map, with smallest values in the interior to larger values toward the outside. Note that the size and color scales are different from map to map. The ellipse at the lower left shows the beam. Left: D/L spectral index map with error contours at $0.01,0.025$, and 0.05 . Center: $\mathrm{C} / \mathrm{L}$ spectral index with error contours at $0.01,0.025$, and 0.05 . Right: $\mathrm{B} / \mathrm{L}$ with error contours at 0.15 and 0.2 (the south-west contour is 0.15 and the broken north contour is 0.2 ).
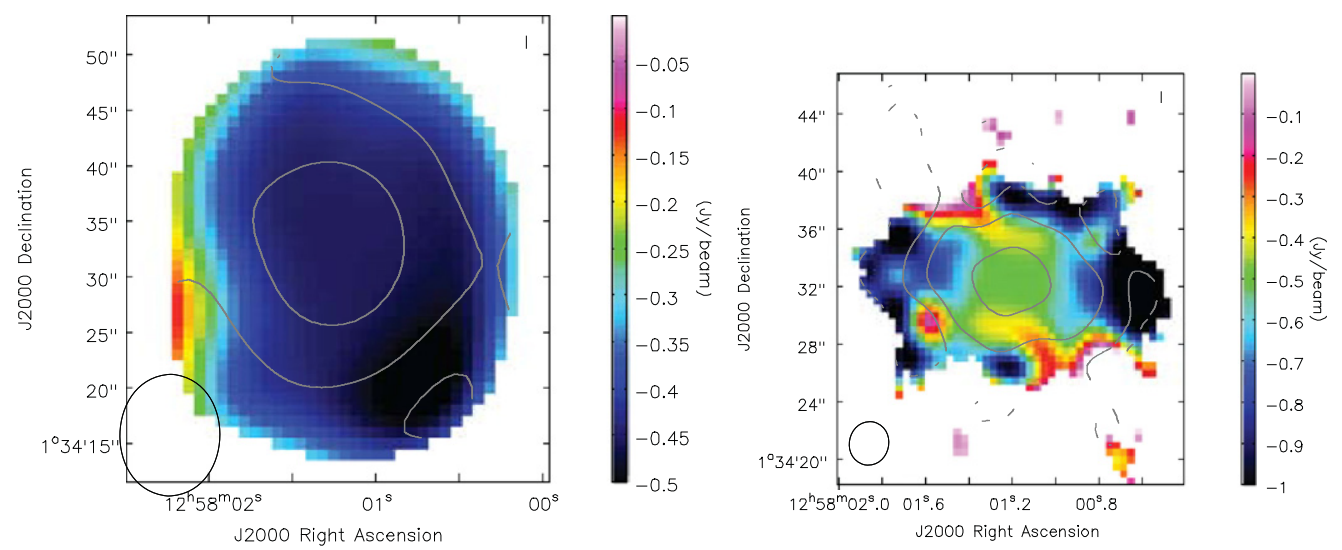

Figure 4. C-band spectral index maps formed as described in Section 2. Colors represent the spectral index with the color legend shown at right. Gray solid contours represent values from the corresponding error map, with smallest values in the interior to larger values toward the outside. Note that the size and color scales are different from map to map. The ellipse at the lower left shows the beam. Left: $\mathrm{D} / \mathrm{C}$ spectral index map with error contours at $0.01,0.05$, and 0.1 . Right: $\mathrm{C} / \mathrm{C}$ with error contours at $0.01,0.1$, and 0.2 .

and should also be the most accurate, given that radio data do not suffer from extinction as do optical data. Nevertheless, our position agrees well with that of the SDSS (uncertainty of 0 ".2) as quoted in NED.

The size of the central core, after deconvolving from the synthesized beam, is also provided. A comparison with the beam sizes given in Table 2 shows that the central core is unresolved, as measured in the highest resolution data sets. (Repeating this exercise for the lower resolution data gives the same conclusion.) The deconvolved source size $(0.2 \rightarrow 0.7$ arcsec) is also of order or smaller than the pixel size $(0.5 \operatorname{arcsec}=41 \mathrm{pc})$, which sets a limit on the possible precision of size measurable by a Gaussian fit. Therefore, the unresolved core could be smaller still.

\subsubsection{In-band Spectral Indices}

Because the source is variable (Section 3.1.4), a "classical" computation of spectral index between L-band and C-band 
would not yield reliable results since the two different bands were measured at different times. Fortunately, the wide bands used in the CHANG-ES observations make it possible to compute in-band spectral indices (Section 2). Figures 3 and 4 show the resulting maps for L-band and C-band, respectively.

Figure 3 shows successively higher resolution (left to right) L-band spectral index maps. A largely north-south gradient is visible in the lower resolution $\mathrm{D} / \mathrm{L}$ and $\mathrm{C} / \mathrm{L}$ images, although not in the high resolution $\mathrm{B} / \mathrm{L}$ data. These gradients are intriguing because they appear to align in the direction of the optical cone (Section 3.1) and are visible in two independent data sets. Since the gradients become most pronounced after self-calibration, a variety of tests were run with different selfcalibration iterations, inputs, and box sizes; nevertheless, the gradient remains. To be cautious, however, we computed the weighted average spectral indices, $\bar{\alpha}$ (see details, Section 2), only for the central FWHM of the emission shown in Figure 3. That is, $\bar{\alpha}$ applies to a size equivalent to the beam size shown at lower left, rather than to the entire region shown in the figures. This also ensures that the spectral indices are measured only in regions where the error (as shown by contours) is lowest and emission is highest. The results are given in Table 3. Note that the L-band spectral indices in all configurations are positive, and of order +1 .

Figure 4 shows the C-band spectral indices. The weighted mean spectral indices, again for the central FWHM of the emission, are given in Table 3. C-band spectral indices are all negative, of order -0.5 .

It is clear that the spectral index of this compact core peaks between L-band $(\nu=1.57 \mathrm{GHz})$ and $\mathrm{C}$-band $(\nu=6.00 \mathrm{GHz})$. The source therefore may be a nearby lower-luminosity analogue of the GPS sources seen at higher redshifts (e.g., O'dea et al. 1991).

The importance of measuring in-band spectral indices cannot be overstated for these observations. Since the source is variable, a classical measurement of the spectral index between the centers of the two bands would give grossly erroneous results, even if the observations had been simultaneous, because a straight line (in log space) would simply have been drawn between the flux densities of the midpoints of the two bands (see also Figure 6). Our in-band spectral indices correspond to the slopes of the spectrum at the midpoints of both bands, a fact that we will exploit in Section 3.1.5.

Finally, the spectral index of the weak disk emission, $\alpha_{\text {disk }}=-0.74$ (Section 3.1.1), is not the same as that of the central core in either band. Since the HPBW region in which $\bar{\alpha}$ was measured for the low resolution data $(\mathrm{D} / \mathrm{L}, \mathrm{D} / \mathrm{C}$, and $\mathrm{C} / \mathrm{L})$ encompasses both the core and the disk, a small correction is required to obtain the spectral index of the core itself. For each band, this was done by determining the flux density of the disk at the upper and lower ends of the band using $\alpha_{\text {disk }}$, determining the total flux density for the upper and lower ends of the band using $\bar{\alpha}$, subtracting off the disk flux density from the total at the two band ends, and recalculating the inband spectral indices, yielding $\overline{\alpha_{\mathrm{AGN}}}$. These results are also given in Table 3.

\subsubsection{Variability}

Table 4 reveals a significant change in flux density with time for NGC 4845.

For the historical data at L-band, the flux density appears to decrease from the 1995 NVSS (D-array) value of 46 mJy to the

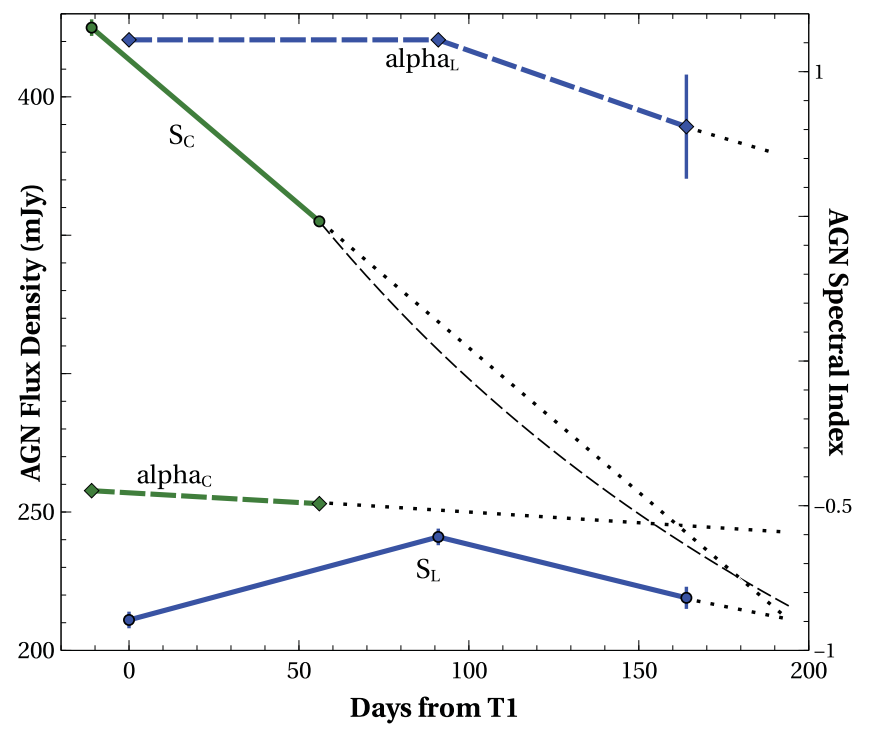

Figure 5. AGN flux densities, $S_{\mathrm{AGN}}$ (solid lines with circular markers), and inband spectral indices, $\bar{\alpha}$ (dashed lines with diamond markers), are shown for the L-band (blue) and C-band (green) data, from Tables 4 and 3, as a function of time, where $T_{1}=2011$ December 30 . Error bars are shown but are mostly smaller than the marker size. Dotted lines show linear extrapolations of the curves to the date, 2012 July $13\left(T_{1}+196\right.$ days), at which the L-band and C-band flux densities are equal (Table 6). The dashed curve shows an extrapolation of the C-band flux density of the form, $S_{\mathrm{C}} \propto t^{-5 / 3}$ (Equation (22)).

1998 FIRST (B-array) value of 34 mJy. However, the FIRST B-array data reveals only a point source without the disk, because the rms noise is of order the brightness of the disk as measured in the CHANG-ES B/L data set. The difference between those two flux densities $(12 \mathrm{mJy})$ may therefore be attributable to some missing low brightness extended emission of the central disk, which we have measured to be $19 \pm 4 \mathrm{mJy}$ (Section 3.1.1). Two additional earlier archival VLA data sets are also available, though unpublished, which we have now reduced. These are L-band D-array data (1988 March 23) and L-band B-array data (1987 November 15) for which the flux densities are 48 and $28 \mathrm{mJy}$, respectively. Given the uncertainties (especially at the earlier epoch), we consider these values to agree with the NVSS and FIRST results, respectively. Therefore, of the limited data available, there was no detected L-band variability prior to 1998 .

On the other hand, our CHANG-ES observations have now shown an increase at L-band of approximately a factor of six compared to the NVSS results. Unfortunately, there is no prior measurement of C-band flux density in the literature. The (1986-1987) Green Bank 4.85 GHz survey (GB6; Gregory et al. 1996), which covered this region of sky to a limit of $\approx 42 \mathrm{mJy}$ (their Figure 2), did not detect the source. Allowing for the $5 \sigma$ requirement for the source to be included in their list suggests that the C-band flux density was $<210 \mathrm{mJy}$ at the earlier epoch, or a factor of at least two lower than our peak $\mathrm{C}$-band measurement. We suspect that the flux density change was much higher, however, given the L-band measurements and the fact that the source is highly absorbed at L-band but not C-band (Section 4.2).

A plot of AGN flux density and in-band spectral indices (both corrected for the disk) as a function of time for the CHANG-ES data is shown in Figure 5. Earlier historical data have been omitted, given the large lapse in time. This plot 
Table 6

Flux Densities and Spectral Indices for Matching Times

\begin{tabular}{|c|c|c|c|c|c|c|}
\hline \multirow[t]{2}{*}{ Date } & \multicolumn{3}{|c|}{ L-band } & \multicolumn{3}{|c|}{ C-band } \\
\hline & $\begin{array}{l}S_{\mathrm{AGN}} \\
(\mathrm{mJy})\end{array}$ & $\begin{array}{l}\text { Frequency } \\
\quad(\mathrm{GHz})\end{array}$ & $\overline{\alpha_{\mathrm{AGN}}}$ & $\begin{array}{l}S_{\mathrm{AGN}} \\
(\mathrm{mJy})\end{array}$ & $\begin{array}{l}\text { Frequency } \\
\qquad(\mathrm{GHz})\end{array}$ & $\overline{\alpha_{\mathrm{AGN}}}$ \\
\hline$T_{2}\left(2012 \mathrm{Feb} 24=T_{1}+56\right.$ days $)$ & 229 & 1.575 & +1.11 & 355 & 5.999 & -0.493 \\
\hline$T_{3}$ (2012 Jul $13=T_{1}+196$ days $)$ & 209 & 1.575 & +0.68 & 209 & 5.999 & -0.587 \\
\hline
\end{tabular}

Note. L-band and C-band flux densities and spectral indices for matching times, interpolated or extrapolated from Figure 5 (see also Section 3.1.4).

Table 7

Parameters of Polynomial Spectral Fits

\begin{tabular}{lccccc}
\hline \hline Date & $a_{0}$ & $a_{1}$ & $a_{2}$ & $a_{3}$ & $\nu_{m}{ }^{\mathrm{a}}$ \\
\hline Time 1 $\left(T_{1}=\right.$ 2011 Dec 30) & -100.27 & 249.79 & -35.17 & 1.305 & 4.87 \\
Time 2 $\left(T_{2}=\right.$ 2012 Feb 24) & -156.93 & 338.22 & -65.22 & 3.845 & 4.03 \\
Time 3 (T, $T_{3}=12$ Jul 13) & -36.78 & 230.71 & -53.02 & 3.566 & 3.22 \\
\hline
\end{tabular}

Note. Parameters of the polynomial fit to the spectrum for three different time stamps. The fit is to $S_{\nu}=a_{0}+a_{1} \nu+a_{2} \nu^{2}+a_{3} \nu^{3}$, where $S_{\nu}$ is the flux density (mJy) at frequency, $\nu(\mathrm{GHz})$, and simultaneously matching the slope of this function to the slope of the power law spectrum, $S_{\nu} \propto \nu^{\alpha}$, in each band (see also Section 3.1.5). Input data are from Table 6.

${ }^{a}$ Frequency of the spectral peak $(\mathrm{GHz})$.

${ }^{\mathrm{b}}$ Flux density at $\nu_{m}$ (mJy).

shows that the AGN flux density has varied even over the fewmonth time period of our observations. At L-band, the flux density increases and then decreases. At C-band, the flux density shows a strong decline. The spectral indices (dashed curves) also reveal variations in both bands.

From these data, we adopt three time stamps, namely Time 1 $\left(T_{1}\right)$ : the first time at which L-band data are obtained and for which C-band data can be interpolated from its curve; Time 2 ( $T_{2}=T_{1}+56$ days): the last time at which C-band data have been obtained and for which L-band data can be interpolated; and Time $3\left(T_{3}=T_{1}+196\right.$ days): an extrapolation of both L-band and C-band data to the time at which the flux densities at both bands are equal. In all cases, simple linear interpolations or extrapolations are performed, and the same is done for the spectral indices. The resulting data (Table 6) provide flux densities and spectral indices for matching times.

\subsubsection{The AGN Spectrum and its Variability}

From the data of Figure 5, it is possible, not only to estimate the spectrum of the AGN, but also to see how it varies with time. For each time stamp, we now have four data points that can be used to constrain the spectrum: the L-band flux density, the L-band spectral index, the C-band flux density, and the C-band spectral index, where the spectral indices represent tangent slopes of the spectrum at the two frequencies. We then fit a polynomial to the spectrum of the form

$$
S_{\nu}=a_{0}+a_{1} \nu+a_{2} \nu^{2}+a_{3} \nu^{3}
$$

where $S_{\nu}$ is the flux density at frequency $\nu$, and $a_{i}$ are constants. The derivative of this equation is equated to the derivative of the flux density equation, $S_{\nu} \propto \nu^{\alpha}$, both of which represent tangent slopes to the spectrum at frequency, $\nu$. With two flux densities and two slopes at each time stamp, Equation (3) was solved for the spectrum at each time stamp.

The results are given in Table 7 and the spectra are plotted in Figure 6. It is now clear that the turnover of the spectral index is shifting to lower frequencies with time, and the peak of the

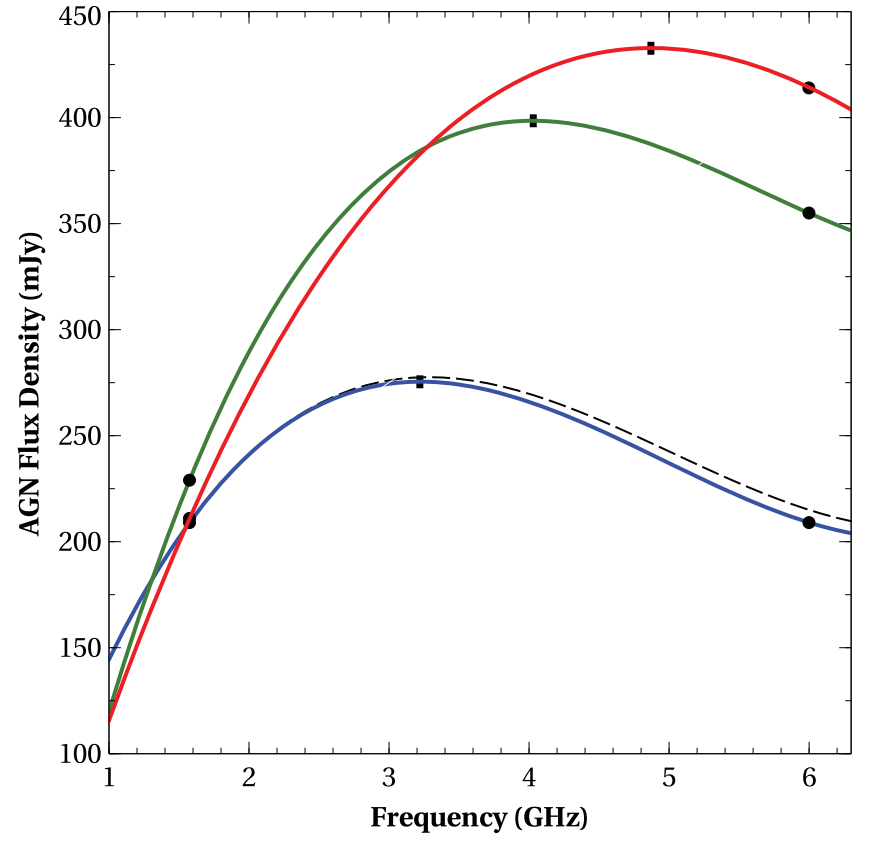

Figure 6. Spectra from polynomial fits (Table 7) for three time stamps: $T_{1}$ (2011 Dec 30) in red, $T_{2}=T_{1}+56$ days in green, and $T_{3}=T_{1}+196$ days in blue. Data (black dots) are from Table 6 and the peak of each curve is marked with a small bar. Note that each curve is an explicit solution to a polynomial fit with four constraints: two flux density points and two slopes. $T_{3}$ data have been extrapolated from the measured data. The black dashed curve corresponds to the non-linear extrapolation shown by the black dashed curve in Figure 5. Measurement error bars are typically smaller than the points.

spectrum also declines with time. The peak frequency shifts from 4.9 to 4.0 to $3.2 \mathrm{GHz}$.

We carried out some tests to verify the validity of this result. For example, the same trend can be seen in the original data prior to subtracting the disk emission. If we repeat this exercise prior to subtracting the disk contribution to both flux density and spectral index, then $\nu_{m}$ is altered by less than $1 \%$ for all 
time stamps, $S_{\max }$ for time steps $T_{1}$ and $T_{2}$ increase by less than $2 \%$, and the peak flux density for time step $T_{3}$ increases by $15 \%$. Given the error bars in the L-band spectral index, we also repeated the analysis assuming that $\alpha_{L}$ has not varied at all (i.e., letting $\overline{\alpha_{L}} \approx 1.1$ for each time stamp); there is a $1 \%$ decrease in the peak frequency, $\nu_{m}$, and a $5 \%$ decrease in the peak flux density, $S_{\max }$, for the $T_{3}$ curve only. Fitting a curve, where possible, instead of simple straight lines (e.g., using a secondorder polynomial for the L-band data of Figure 5), also does not alter the above conclusions.

The final issue is related to extrapolation/interpolation. Note that point $T_{1}$ is measured at L-band and interpolated at C-band; point $T_{2}$ is measured at $\mathrm{C}$-band and interpolated at $\mathrm{L}$-band. Only point $T_{3}$ is an extrapolation in time. If we instead make a more modest extrapolation (i.e., $T_{1}+100$ days, or 44 days after the last measured C-band point, but still an interpolation at Lband), then the trend is still consistent with what is shown in Figure 6, again with a declining peak that is shifting to lower frequencies with time. This intermediate curve (not shown) falls between the $T_{2}$ and $T_{3}$ curves of Figure 6 .

At time $T_{3}$, the L-band extrapolation is small, both when one considers the elapsed time (32 days after the last L-band measurement) as well as the modest implied variations in flux density and spectral index. It is the C-band flux density that has undergone the largest change as a result of our extrapolation (Figure 5). We have made the simplest possible assumption as to the form of its time decay (i.e., linear), arguing that to impose anything more complex (and arbitrary) is not justified by the data.

However, in anticipation of our model results (see Section 4.3), we can also try an $S_{\nu} \propto t^{-5 / 3}$ dependence (Equation (22)) on the C-band flux density. The results are shown as the black dashed curves in Figures 5 and 6 . Note that we restrict this curve only to the region from $T_{2}$ to $T_{3}$ in which a true extrapolation at C-band is needed. In addition, the curve's time dependence applies to the highly optically thin regime, whereas there is actually a changing optical depth with time, as explained in Section 4.3 (hence the "disjoint" slopes at $T_{2}$ ). This new extrapolation increases the C-band $T_{3}$ flux density by only $3 \%$ from $209 \mathrm{mJy}$ (Table 6) to $215 \mathrm{mJy}$, and its affect on the behavior of the curves in Figure 6 is minor.

Any other more dramatic extrapolation is both unknown and speculative and, as stated above, even modest extrapolations still follow the trends shown in Figure 6. In summary, we conclude that the behavior of declining flux densities and frequencies with time is a robust conclusion within the constraints of the available data.

Fitting the spectrum with a polynomial is the best and most accurate way of describing the source's variable behavior with time, as observed. It does not assume any physical model, however, which is an issue we address in Section 4.

\subsubsection{The Absence of Large-scale Disk Emission}

No extended disk emission beyond the $1.8 \mathrm{kpc}$ diameter central disk seen in Figures 1 and $2(\mathrm{~B} / \mathrm{L}$ and $\mathrm{C} / \mathrm{C}$ data, respectively) has been detected from NGC 4845. This does not appear to be a sensitivity or DR problem when compared to other galaxies in the CHANG-ES sample (Wiegert et al. 2015). In addition, measurable emission is seen in NGC 4845 throughout the large-scale disk in all Wide-field Infrared Survey Explorer (IR) images, as well as in Galaxy Evolution Explorer (UV) images. Previous radio continuum observations
(Table 2) similarly reveal radio emission that is spatially confined to the central region of the galaxy. A thorough discussion of this issue, for example, a study of star formation (SF) rates and timescales in comparison to the radio emission, must await a future paper. We do note, however, that truncated gaseous disks and galaxy stripping are well-known properties of some Virgo Cluster galaxies (e.g., Cayatte et al. 1990; Kenney \& Koopmann 1999; Kenney et al. 2004; Murphy et al. 2009; Vollmer et al. 2013).

\subsection{Polarized Emission}

Flux density measurements for linear polarization, $P$, circular polarization, $V$, and their fractions of the total unpolarized flux density, $S$, are given in Table 4 . In all arrays and frequencies, the percentage linear polarization is less than or of order $0.5 \%$. Since the polarization calibration cannot be guaranteed to be better than $0.5 \%$ (S. Myers 2015, private communication), ${ }^{12}$ we cannot claim to have detected any linear polarization in any configuration/band combination for NGC 4845.

On the other hand, there is a clear measurement of circular polarization at L-band, although not at C-band. The circular polarization is quite strong, with a flux density of 5.5-6.6 mJy

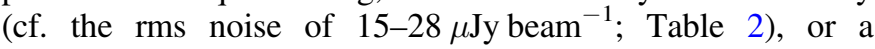
polarization percentage of $2 \%-3 \%$. Significantly, each independent observation has resulted in approximately the same percentage circular polarization.

It is important to consider whether the circularly polarized emission could have been artificially introduced. For example, any errors will affect a $V$ signal more strongly than total intensity because $V$ is measuring a difference between correlated signals. ${ }^{13}$ It is well known that the VLA suffers from "beam squint," which can result in spurious signals offaxis (Brisken 2003). The center of NGC 4845, however, was placed at the field center, where beam squint is zero. Selfcalibration can also result in spurious $V$ signals if there are other off-axis circularly polarized sources in the field (see Homan \& Wardle 1999, for details of a related example).

Our best counterexample, however, is the $\mathrm{B} / \mathrm{L}$ data for which negligible signal was observed other than in the center of the map. This data set was re-imaged with no self-calibration table applied, and (although beset with higher residual sidelobes due to the lack of self-calibration) the resulting image still shows a significant $V$ signal. An alternative selfcalibration method was also employed ${ }^{14}$; again, the result was poorer with higher sidelobes, but there was no change in the $V$ flux density. Imaging of the primary calibrator, 3C 286, showed $V / I<0.17 \%$ and the polarization calibrator, OQ 208, had $V / I<0.08 \%$.

In summary, the circular polarization appears to be of order a few percent in $V / S$ and also $V / S_{\text {AGN }}$ (Table 4). Moreover, we find the $V$ spectral index, $\bar{\alpha}_{V}$, again possible to determine because of our broad bands (Section 2), to be quite steep, of order $-2 \rightarrow-3.5$ (Table 3 ). Given the above discussion, we do not claim that the small variations between arrays are significant. The rather striking result of measurable circular

\footnotetext{
12 See also the NRAO polarimetry online documents.

${ }^{13} V=(\mathrm{RR}-\mathrm{LL}) / 2$, where RR refers to the correlation of "right-right"-hand polarization and LL refers to "left-left."

14 A "T" type self-calibration in which a single solution for both polarizations is obtained.
} 


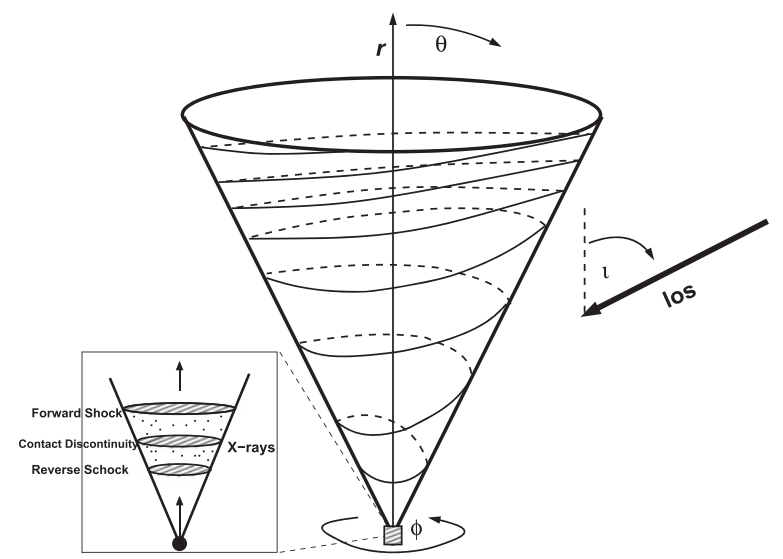

Figure 7. One side of an outflow, of order $0.1 \mathrm{pc}$ in size, which forms a wideangle cone $(\theta$ large) or narrow angle jet (small $\theta$ ) of relativistic electrons. The origin of the outflow is at the apex and the helical curve represents the azimuthal magnetic field. A suggested line of sight (LOS), which passes through a sheath of material in or around the cone, is shown by the heavy back arrow, which makes an angle, $\iota$, with the radial (along the cone axis) direction. A depolarizing screen (not shown) is outside the cone along the LOS. Inset: blow-up of the apex region, of order $1000 \mathrm{AU}$ in size, showing the shocked region in which $\mathrm{X}$-rays are generated.

polarization and its variation with frequency will be discussed further in Section 4.4.

\section{DISCUSSION}

In this section, we seek to explain the following observations: a) the turnover in the radio spectrum and its evolution with time (both amplitude and frequency shifts), conveniently summarized for this "GPS" source in Figure 6; and b) the detection of circular polarization while a believable linearly polarized signal is absent. We also attempt to connect the X-ray observations of Nikolajuk \& Walter (2013) with our radio data.

We describe more generally the jet/cone model with basic geometry that lies behind our general thinking in Appendix A. For this section, it is sufficient to imagine a source of relativistic particles as illustrated in the cone geometry of Figure 7. The optical cone observed in Figure 2 suggests such a geometry on larger scales, possibly representing an earlier outflow. As we do not know the small-scale opening angle, however, we use "jet" or "cone" interchangeably when referring to our model.

We are aided by the fact that the X-ray outburst was observed and provides a timescale. We will refer to the time of the peak of the X-ray light curve (2011 January 22) as $t_{0}$ (the disruption of a mass that apparently triggered the X-ray flare occurred 60-100 days earlier; Nikolajuk \& Walter 2013). Our radio data span six months (Table 2) and the first time stamp from our spectral fits (Table 7 ) occurs at $T_{1}=342$ days $\approx 1$ year after the X-ray peak.

Several reasonable simplifications can be made. First, for a very fast jet, transformations would be required between the source and observer's rest frame for parameters such as jet angle, brightness, magnetic field, and so on. For completeness, these transformations are provided in Appendix B; however, we find that unless the bulk jet speed is ultrarelativistic, such transformations are not required and will not be applied here. Second, our calculations are order of magnitude estimates to test for feasibility and consistency with the observations.

\subsection{Source Size}

From observations, the core of NGC 4845 is unresolved, likely less than 0.5 arcsec (Section 3.1.2), so we do not have sufficient resolution to measure its size, $\theta_{s}$, directly. However, the variability itself suggests a limiting size. If the source was created with the X-ray flare observed by INTEGRAL in 2011 January (Nikolajuk \& Walter 2013), then the projected physical size should be $\leqslant c t \sin (\iota) \approx 10^{18} \sin (\iota) \mathrm{cm}$, where $\iota$ is the inclination of the major source axis to the line of sight (Figure 7 ) and $t$ is the elapsed time. For $t \approx 1$ year (cf. dates of Table 2) and a distance of $17 \mathrm{Mpc}$ (Section 1), this suggests that $\theta_{\mathrm{s}} \leqslant 4 \sin (\iota)$ mas. Thus if $\iota=0^{\circ}$, we are looking down the major axis of the cone along the outflow and $\theta_{s}$ would relate to the cross-sectional diameter of the cone; if $\iota=90^{\circ}$, the cone is aligned with the sky plane and $\theta_{s}$ would relate to a typical cone size in projection. In the following, we adopt $\theta_{s} \sim 1$ mas $\left(\sim 0.1 \mathrm{pc}\right.$ or $\left.\approx 10^{17} \mathrm{~cm}\right)$ as a fiducial value.

Note that the brightness temperature at $20 \mathrm{~cm}$ is $\approx 3.4 \times 10^{11}$ $\mathrm{K}$ if the source size is of order 1 mas. This is still below the limit for catastrophic inverse Compton losses. GPS sources at higher redshift suggest a range of sizes with the lowest $\approx 1 \mathrm{pc}$ (Fanti 2009), hence the core of NGC 4845 extends the array of GPS source sizes an order of magnitude smaller.

\subsection{The Low Frequency Spectral Turnover}

A consistently difficult problem in the past has been to understand whether low frequency turnovers are caused by thermal absorption or synchrotron self-absorption (SSA). In this section we explore both possibilities and conclude that the dominant cause of the turnover is likely SSA.

We first, however, exclude Razin suppression of the low frequency emission as the cause of the turnover because the predicted exponential cutoff below the Razin frequency (Melrose 1980) was not observed. In addition, at least for a homogeneous source, the effect does not give a dependence of circular polarization on frequency, which we actually detect (Section 3.2).

\subsubsection{Synchrotron Self-absorption}

For SSA as an explanation for a GPS source (see, e.g., Artyukh et al. 2008) one expects the brightness temperature of the source to approach the electron kinetic temperature (e.g., Longair 1994). The source brightness temperature in terms of the wavelength, $\lambda$, and flux density, $S_{\nu}$, is

$$
T_{b}=\frac{\lambda^{2}}{2 k} \frac{S_{\nu}}{\Omega_{\mathrm{s}}} \mathrm{K} \approx \frac{7 \times 10^{7} \lambda_{6}^{2}}{\theta_{\mathrm{s}}(\mathrm{mas})^{2}} S_{\nu} \mathrm{K}
$$

where $\Omega_{\mathrm{s}}=(\pi / 4) \theta_{\mathrm{s}}^{2}, \theta_{s}$ is the source size, and $k$ is Boltzmann's constant. We have taken $\lambda$ in units of $6 \mathrm{~cm}, \theta_{s}$ in mas, and the flux density in mJy. The kinetic temperature, $T_{e}$, for relativistic electrons is given by $k T_{e}=\gamma m_{e} c^{2} / 3$. Equating these two temperatures gives the limiting selfabsorbed flux density at a peak frequency for electrons of energy, $\gamma$, as

$$
S_{\nu}=\sqrt{\frac{2}{3}} \frac{\pi}{3} \frac{m_{e} \theta_{\mathrm{s}}^{2}}{\nu_{\mathrm{g}}^{1 / 2}} \nu^{5 / 2} \approx 950 \frac{\nu_{5}^{5 / 2} \theta_{\mathrm{s}}(\mathrm{mas})^{2}}{B_{\perp}(-2)^{1 / 2}} \mathrm{mJy} .
$$

where $\nu_{\mathrm{g}} \equiv e B_{\perp} /(2 \pi) m_{\mathrm{ec}}$ is the gyrofrequency, $e$ and $m_{e}$ are electronic properties, and $\nu_{5}$ is the frequency in units of $5 \mathrm{GHz}$. 
From this last expression we can infer the universal (thermodynamic) self-absorbed spectral index, $\alpha=2.5$, and declining flux densities with decreasing frequency.

Adopting $B_{\perp}(-2)=1$ (see Appendix E) and $\theta_{s(\operatorname{mas})=1}$ (Section 4.1), the observed flux density at $6 \mathrm{~cm}$ is lower than the flux density given by Equation (5), indicating that selfabsorption is not complete at this frequency. However, at $20 \mathrm{~cm}$, the observed flux density is much higher than that of Equation (5) (by an order of magnitude). In other words, the observed flux density at $6 \mathrm{~cm}$ implies a peak (turnover) frequency below $6 \mathrm{~cm}$, whereas the observed flux density at $20 \mathrm{~cm}$ implies a peak frequency well above $20 \mathrm{~cm}$. Thus, we expect our results summarized in Figure 6 to describe a spectral region that is transiting toward self-absorption at the low frequencies.

One of the principal results of these observations is the measurement of the in-band spectral indices, which have been used to define the fits of Figure 6. If we are to explain these curves as a transition to SSA, then we must consider the rollover in spectral index from an optically thin spectral index of $\alpha=(1-p) / 2 \approx-0.5 \Rightarrow p=2$ (Table 3) (using $N(E)=N_{o} E^{-p}$ for the energy distribution function of the electrons) toward the index $\alpha=2.5$, which is expected when the source is fully self-absorbed, as shown above. This rollover is described in (Pacholczyk 1970, p. 97). We calculate

$$
\alpha=\frac{d \ln J(z)}{d \ln z},
$$

where

$$
J(z)=z^{5 / 2}\left(1-\exp \left(-z^{-(2+p / 2)}\right)\right),
$$

and $z \equiv \nu / \nu_{1}$. The frequency at which the optical depth equals one is $\nu_{1}$; for $p=2$ this is given by $\nu_{1}=0.707 \nu_{\mathrm{c}}$, with $\nu_{\mathrm{c}}$ being the critical frequency. The result is shown in Figure 8 for several values of $p$. The reference case for our purposes is the lower curve at large $z$ for which $p=2$. We see that $\alpha$ varies from the optically thin value of -0.5 to a moderately optically thick value of $\alpha=+1.0$ (as observed at L-band) over roughly a factor three in frequency. However because of the steepness of the rising curve at $z=1$, it is possible to have $\alpha$ in the optically thick region ranging from just below 0.5 to 1.0 over essentially the same range in frequency. This is a reasonable explanation of the curves in Figure 6 between 6 and $20 \mathrm{~cm}$ (C-band and L-band, respectively). Given the index $p$, we emphasize that the only free parameter is $\nu_{1}$ in describing the spectral shape. To detect the fully self-absorbed spectral index, one has to descend another factor of two in frequency. A fit by the synchrotron model to all the data is included in Figure 9.

We can also estimate the relativistic electron density required to produce the turnover. The absorption coefficient for the synchrotron particles is approximately

$$
\kappa_{\mathrm{er} \nu}=1.7 \times 10^{-14} N_{o} \frac{B_{\perp}(-2)^{2}}{\nu_{1}^{3}} \mathrm{~cm}^{-1},
$$

where the subscript, er, refers to relativistic electrons and $\nu_{1}$ is in units of GHz. When $p=2$, the coefficient $N_{o} \approx n_{\mathrm{er}} E_{o}$. Here $n_{\mathrm{er}}$ is the relativistic electron density and $E_{o}=\gamma_{o} m_{e} c^{2}$ is the lower energy cutoff of the distribution. If $L$ measures the line of sight distance through the source then we conclude, by setting

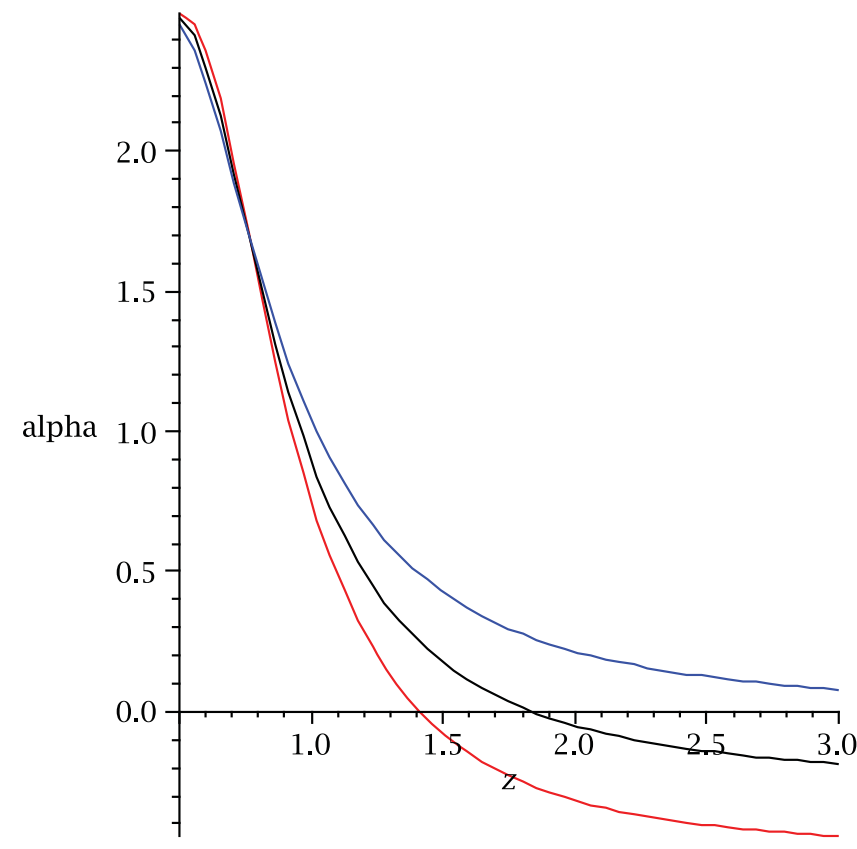

Figure 8. Rollover in the spectral index during the onset of synchrotron selfabsorption at low frequencies. The frequency is measured in units of $\nu_{1}$, the frequency at which the optical depth is 1 (i.e., $z=\nu / \nu_{1}$ ). This is 0.707 times the peak frequency for $p=2$. The lower curve at large frequency is our reference case for which $p=2(\alpha=-0.5)$. The higher curves at large frequency taken in rising order are for $p=1.5$ and $p=1$, respectively. The transition from thin to moderately thick is over roughly a factor of three in frequency. Another factor of two in frequency is required to attain the completely self-absorped index.

the optical depth equal to one, that

$$
n_{\mathrm{er}} L=2 \times 10^{19} \frac{1}{\gamma_{o}(10) B_{\perp}(-2)^{2}} \mathrm{~cm}^{-2} .
$$

Here $\gamma_{o}(10)$ is the lower cutoff in units of 10 (which we set to 1 , following other authors) and we have taken $\nu_{1}=1.5$. Nominally this suggests, given our proposed source size $\left(10^{17}\right)$, that $n_{\mathrm{er}} \approx 200 \mathrm{~cm}^{-3}$.

If the number of protons is the same, then a rough calculation of mass for a cylinder (approximating a cone) of length and diameter, $10^{17} \mathrm{~cm}$, would result in $\sim 3 \times 10^{29} \mathrm{~g}$ (or more if thermal gas is entrained). This is $5 \%-10 \%$ of the mass of $3 \times 10^{30} \leqslant M \leqslant 6 \times 10^{30} \mathrm{~g}$ estimated by Nikolajuk \& Walter (2013) to have produced the X-ray flare via accretion of a "super-Jupiter" object. In the latter case, the authors suggest that $10 \%$ of a "super-Jupiter" of mass, 14-30 Jupiter masses, has produced the X-ray flare. This suggests some consistency between the relativistic electron density required to explain the radio spectral turnover and the expected quantity of particles that are supposed to be involved in the X-ray event (Figure 7).

For a more thorough investigation of SSA as a possible cause of the turnover, we also fit the $T_{2}$ time stamp with a pure SSA spectrum, allowing $\nu_{1}, p$, and the amplitude to freely vary. For the latter, we introduce a scaling factor, $\tilde{C}$, which converts the amplitudes to a flux density, $S(z)$, for comparison to the data, that is, $S(z)=\tilde{C} J(z)$. The best fit is chosen by minimizing $\chi^{2}$ during a least squares fit.

The resulting best-fit parameters are given in Table 8 and the spectrum, along with its derivative, are plotted as the red curves 

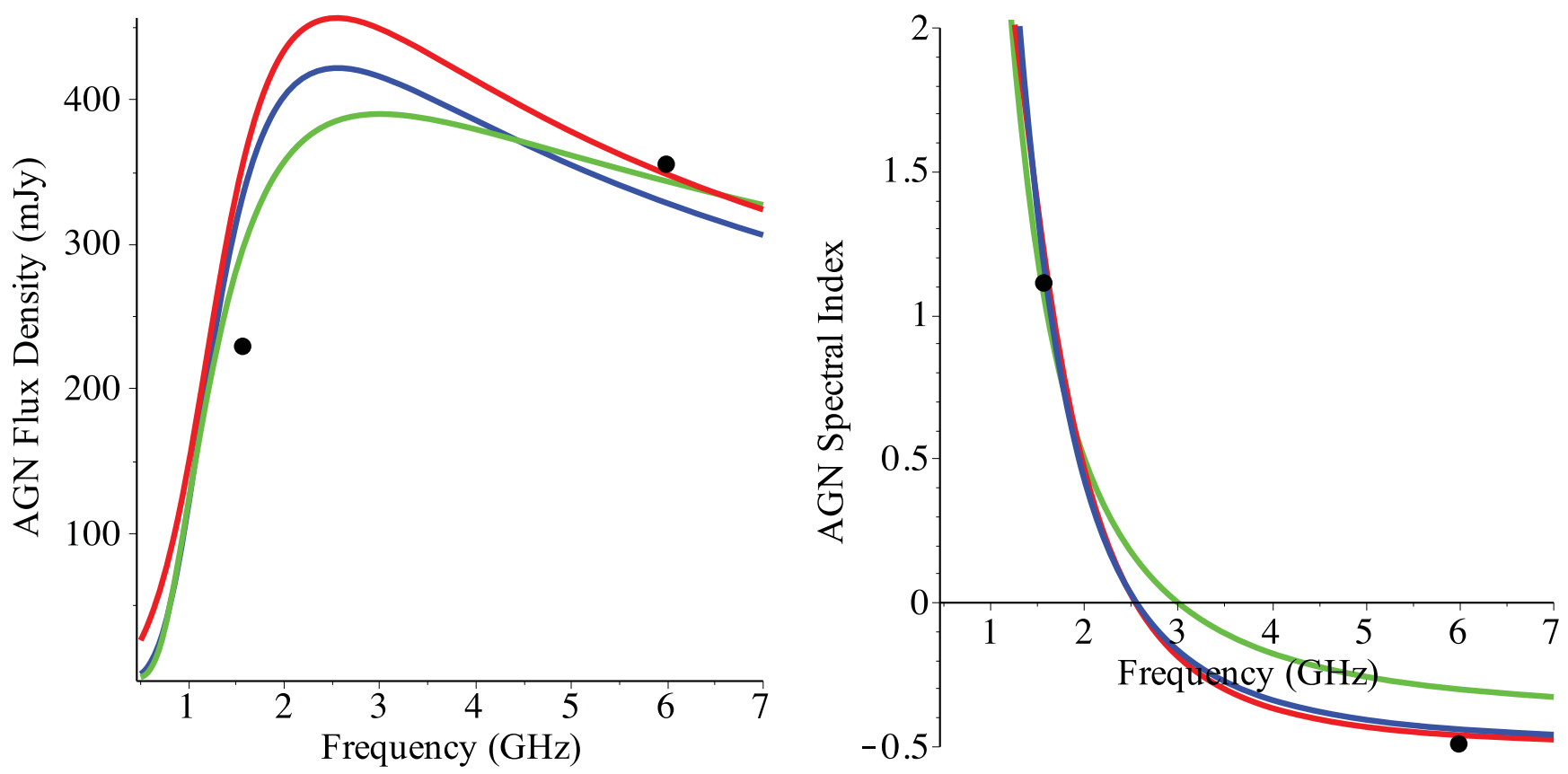

Figure 9. Best fit synchrotron-self-absorption-only spectrum (red), thermal-absorption-only (green), and a synchrotron self-absorption plus a thermally absorbing screen (blue), all as applicable to time, $T_{2}$. Best fit parameters are provided in Table 8. Black dots represent the data. The left plot shows the spectra, displaying fits to the flux densities, whereas the right plot shows the slopes, displaying fits to the spectral indices. Measurement error bars are typically smaller than the points.

Table 8

Parameters of Synchrotron and Thermal Spectral Fits at $T_{2}$

\begin{tabular}{lcccccccc}
\hline \hline & $p$ & $\nu_{1}$ & $\tilde{\kappa}$ & $\tilde{C}$ & $S_{\mathrm{L}}$ & $S_{\mathrm{C}}$ & $\alpha_{\mathrm{L}}$ & $\alpha_{\mathrm{C}}$ \\
\hline Synchrotron $^{\mathrm{a}}$ & 2.0 & 1.8 & & 645 & 358 & 349 & 1.20 & -0.460 \\
Thermal $^{\mathrm{b}}$ & 1.8 & & 1.8 & 740 & 299 & 344 & 1.05 & -0.300 \\
Synchrotron $^{2}$ Thermal $^{\mathrm{c}}$ & 2.0 & 1.5 & 0.3 & 675 & 336 & 329 & 1.16 & -0.439 \\
Data $^{\mathrm{d}}$ & $\cdots$ & $\cdots$ & $\cdots$ & $\cdots$ & 229 & 355 & 1.11 & -0.493 \\
\hline
\end{tabular}

Notes. Best fit parameters for the various fits as described in Sections 4.2 and Figure 9. Subscripts L and C refer to the L-band and C-band, respectively. $\chi^{2}$ is a measure of the goodness of fit, here defined as $\chi^{2}=\left\{\left[S_{\mathrm{L}}(\text { fit })-S_{\mathrm{L}}(\text { data })\right]^{2}+\left[S_{\mathrm{C}}(\text { fit })-S_{\mathrm{C}}(\text { data })\right]^{2}\right\} /(\Delta S)^{2}+\left\{\left[\alpha_{\mathrm{L}}(\text { fit })-\alpha_{\mathrm{L}}(\text { data })\right]^{2}+\right.$ $\left.\left[\alpha_{\mathrm{C}} \text { (fit) }-\alpha_{\mathrm{C}} \text { (data) }\right]^{2}\right\} /(\Delta \alpha)^{2}$, where we have taken $\Delta S=5 \mathrm{mJy}$ and $\Delta \alpha=0.01$ as representative values. Note that $\tilde{C}$ is defined differently, depending on the fit (see Section 4.2).

${ }^{a}$ Low-frequency turnover due to synchrotron self-absorption only.

$\mathrm{b}$ Turnover due to thermal absorption only.

c Turnover due to synchrotron self-absorption plus a foreground thermal screen.

${ }^{\mathrm{d}}$ Data are from Table 6.

in Figure 9. These curves correspond to the generic red curve in Figure 8 when $\nu_{1}=1.8 \mathrm{GHz}$.

\subsubsection{Thermal Absorption}

In this section we consider the synchrotron emission to be optically thin and the turnover to be due to thermal absorption only.

In the simplest approach, because we know the optically thin spectral index and take it to be $\alpha \approx-0.5$ (Table 3), we can compute the spectral index in the optically thick $(\tau=1)$ regime that would result from thermal absorption. In Appendix D we show that purely thermal absorption can not provide a satisfactory fit to both the low frequency and high frequency spectral indices. Improving the fit at one end by adjusting the optical depth causes the fit to become much poorer at the other end.

Just as for the synchrotron model, a more systematic treatment of thermal absorption is to solve for the spectrum as well as its derivative by finding the parameters of best fit. To achieve this, we also allow the synchrotron energy spectral index, $p$, to vary freely; as before, this fixes the optically thin synchrotron spectral index, $\alpha=(1-p) / 2$. The amplitude scaling, $\tilde{C}$, incorporates $C$ of Equation (55), as well as a term to convert from specific intensity to flux density for comparison to observations. Finally, we combine the remaining unknown parameters into a single free parameter, $\tilde{\kappa}$, which is a variant of Equation (54) that also includes the line of sight distance, $s_{17}$ (in units of $10^{17} \mathrm{~cm}$ ), and the frequency at which the synchrotron spectrum becomes optically thick, $\nu_{1}(9)$, in GHz. That is

$$
\tilde{\kappa}=\frac{n_{e c}(4)^{2} s_{17}}{\nu_{1}(9)^{2} T_{4}^{3 / 2}}
$$

where $n_{\mathrm{ec}}(4)$ is the thermal electron density in units of $10^{4} \mathrm{~cm}^{-3}$ and $T_{4}$ is the "cold" electron temperature in units of $10^{4} \mathrm{~K}$. 
The resulting best-fit parameters are listed in Table 8 and shown as green curves in Figure 9. This fit is demonstrably poorer than that of pure SSA, consistent with our statements above. Note particularly the poor fit to the spectral index in C-band.

\subsubsection{Synchrotron Spectrum with a Foreground Thermal Screen}

It is natural to imagine thermal absorption ocurring in a sheath of mixed cold and hot particles surrounding the synchrotron jet, given the lack of linear polarization that we observe (see Appendix E). In principle this could be a dynamic sheath of cold gas entrained (i.e., mixed) with the jet particles, or it could be be a stationary surrounding cloud.

When we continue to assume that the synchrotron emitting particles are additionally self-absorbed, the equations of the fit are

$$
\begin{gathered}
S_{\nu}=\tilde{C} z^{2.5}\left(1-\exp \left(-\frac{1}{z^{q}}\right)\right) \exp \left(-\frac{\tilde{\kappa}}{z^{2}}\right) \\
\alpha=2.5+\frac{2 \tilde{\kappa}}{z^{2}}-\frac{q}{z^{q}} \frac{\exp \left(-\frac{1}{z^{q}}\right)}{\left(1-\exp \left(-\frac{1}{z^{q}}\right)\right)} .
\end{gathered}
$$

We take $q \equiv(2+p / 2), z=\nu / \nu_{1}$ as before, and $\tilde{\kappa}$ as above. This model has four free parameters $p, \nu_{1}, \tilde{\kappa}$, and the amplitude $\tilde{C}$. The results are given in Table 8 and by the blue curve in Figure 9.

The fit is no better than that of SSA alone, although it is equally good. The high frequency spectral index problem of the pure thermal absorption has been removed. However this was accomplished by almost completely removing the thermal absorption at $\mathrm{C}$-band $\left(\propto e^{-\frac{0.3}{16}}\right)$, which leaves the optically thin synchrotron emission unchanged. Nevertheless this is an acceptable configuration.

We observe also from Figure 9 that any of these simple models fails to reproduce the fluxes to observational accuracy. This is not too surprising because we ignored any real geometrical structure in the source. For example, we see from the definition of $\tilde{\kappa}$ that the line of sight required for a given absorption is rather sensitive to $T_{4}$. Moreover, a detailed radiative transfer calculation in the style of Jones (1988) for a given (partially optically thick) geometry may be required.

It is interesting to note that Wang et al. (2014) have invoked very similar ideas to our own regarding SSA and SSC (Synchrotron Self-Compton) in the description of a more distant TDE (Sw J16449.3+573451). Their geometry requires two nested conical jets of different speeds and total energies. The slower outer jet may be compared to our idea of a dynamic sheath. However, we differ from these authors in using the jet to produce the early X-ray emission in a shock lobe followed by the radio emission after the decelerated jet has expanded.

A detailed study is justified for their source because much more data in the x-ray and radio is available than for NGC 4845. For this reason we adopt our simplest model with the smallest number of parameters, namely pure SSA, in the next section on variability. Subsequent more detailed work after future very long baseline interferometry (VLBI) obervations may be justified.

\subsection{Variability of the Spectrum}

\subsubsection{A Single Injection of Particles Followed by Fading Does Not Fit the Data}

In a paper discussing a distant GPS source (Orienti et al. 2010), it was concluded that the overall source spectrum was best explained by an ensemble of electrons in which acceleration had ceased. The authors called this the "fader" phase. Their extensively sampled spectra were fit in terms of an "injection spectral index" and in terms of the ratio of the acceleration cutoff time to the synchrotron lifetime at peak frequency.

In the most successful fit to the overall spectrum (i.e., with a reasonable injection spectral index of $\alpha \approx-0.7$ ) they found a cutoff time that was about $20 \%$ of the synchrotron lifetime of several thousand years. However, these authors did not observe a fresh outburst in this source, so the expected dependence on time could not be checked. This is the distinct advantage of detecting the combined X-ray and radio emission of the outburst in NGC 4845.

We show in Appendix $\mathrm{C}$ that the time evolution of the spectra shown in Figure 6 does not match this simple picture.

\subsubsection{Spectral Variability as a Result of Adiabatic Expansion}

We now wish to understand the time evolution of the source spectrum, again summarized in Figure 6 (from epochs $T_{1}$ to $T_{3}$ ), which we take to be the best representation of the data. As noted, we assume SSA only to minimize the number of free parameters and provide a fit to the observations that is equally as good as a synchrotron spectrum with an additional thermal screen. We therefore suppose the energy spectrum, $N(E)$, of the relativistic particles to be evolving due to energy loss through adiabatic expansion.

This mechanism presupposes pressure equilibrium with the medium surrounding the evolving source against which the relativistic particles are working. This medium may be thermal gas in a "sheath" around the source, perhaps combined with (or indeed dominated by) the "hoop stress" of a helical magnetic field. Such a local confining magnetic field must be created by an externally driven current.

During adiabatic expansion, the equation for the total electron density per unit volume and per unit energy, $E$, $n(\boldsymbol{r}, E, t)$, is (ignoring acceleration, diffusion, and particle source terms)

$$
\partial_{t} n+\nabla \cdot(n v)=\frac{1}{3}(\nabla \cdot v) \partial_{E}(n E) .
$$

where $\boldsymbol{r}$ is a position vector, $\boldsymbol{v}$ is a velocity, and $t$ is time. For our purposes, we integrate this over an expanding volume that contains the same total number of particles at any given time, in order to get the familiar "diffusion-loss" equation (e.g., Longair 1994) for the number of particles per unit energy interval, $N$

$$
\partial_{t} N(E, t)=\frac{1}{3}(\nabla \cdot v) \partial_{E}(N E)
$$

We have assumed that the velocity divergence can be expressed wholly as a function of time (including a constant) in order to perform the spatial integration.

For simplicity (but see Appendix A) we assume that the source expands with the uniform radial velocity, $v_{o} \widehat{\boldsymbol{e}}_{r}$, where 
the radial direction, $r$, is along any generator of a cone. Hence $\nabla \cdot v=2 v_{o} / r=2 / t$, since $r=v_{o} t$. The solution for $N(E, t)$ is then found (e.g., by the method of characteristics) to be

$$
N(E, t)=N_{o} E^{-p}\left(\frac{t}{t_{1}}\right)^{(2(1-p) / 3)} .
$$

Here, the reference time, $t_{1}=342$ days (elapsed time from the $\mathrm{X}$-ray peak at $t_{0}$ ), occurs at the time stamp, $T_{1}$. The energy spectrum at time $t_{1}$ has clearly been taken as usual to be $N$ $(E)=N_{o} E^{-p}$, and subsequently the spectrum is unchanged. But now, when $p=2$,

$$
N_{o}(t)=N_{o}\left(t_{1}\right)\left(t / t_{1}\right)^{-2 / 3} .
$$

The frequency at synchrotron optical depth one $\nu_{1}$ (which is proportional to the peak frequency, $\nu_{m}$ ) satisfies (Pacholczyk 1970)

$$
\nu_{1} \propto N_{o}(t)^{1 / 3} B_{\perp}^{2 / 3} s^{1 / 3},
$$

when $p=2$. If we suppose that the line of sight size, $s \propto t$ (as does $r$ ), and use the form $N_{o}(t)$ found above, then

$$
\nu_{1} \propto\left(t / t_{1}\right)^{1 / 9} B_{\perp}^{2 / 3} .
$$

We now explain the ratios of the peak frequencies, which, from Table 7, are $\nu_{m}\left(T_{1}\right) / \nu_{m}\left(T_{2}\right)=4.87 / 4.03 \approx 1.2$ and $\nu_{m}\left(T_{1}\right) / \nu_{m}\left(T_{3}\right)=4.87 / 3.22 \approx 1.5$ in terms of this expansion. If $B_{\perp} \propto 1 / s$, so that the azimuthal field dominates (the azimuthal field wraps around the cone/jet; Figure 7), then we calculate the ratios to be $(1+56 / 342)^{5 / 9} \approx 1.1$ and $(1+196 / 342)^{5 / 9} \approx$ 1.3 , respectively. If, at the other extreme, we suppose that the radial field dominates $B_{\perp}$, then $B_{\perp} \propto 1 / s^{2}$ and we calculate the ratios to be $(1+56 / 342)^{11 / 9} \approx 1.2$ and $(1+196 / 342)^{11 / 9} \approx$ 1.7 , respectively. Overall, either decline in magnetic field approximately fits the data; however, we show in the following that $B_{\perp} \propto 1 / s^{2}$ is required to explain the amplitude variations. This suggests a rather losely wound, conical magnetic helix together with moderate particle pitch angles in the source.

Finally, we wish to consider the changing amplitudes of these peaks. The specific intensity at the peak frequency, $\nu_{m}$, is given by (Pacholczyk 1970, p. 97)

$$
I_{\nu_{m}} \propto B_{\perp}^{-1 / 2} \nu_{1}^{5 / 2}
$$

Applying Equation (17), this becomes

$$
I_{\nu_{m}} \propto\left(N_{0}(t)\right)^{5 / 6} B_{\perp}{ }^{7 / 6} s^{5 / 6} \propto t^{-37 / 18} .
$$

The latter time dependence results from the fact that each of the above terms can be expressed as a function of time using Equation (15), $B_{\perp} \propto 1 / s^{2}$, and taking a constant velocity so that $s \propto t$.

It remains to convert from specific intensity to the observed flux density, which requires considering how the projected area of the source varies with time. For spherical outflow, this dependence would be $\theta_{\mathrm{s}}{ }^{2} \propto s^{2} \propto t^{2}$. However, a jet/cone geometry should have $v_{r} \gg v_{\perp}$, where $v_{r}$, and $v_{\perp}$ are the velocities along the axis of the cone (the radial direction) and perpendicular to it, respectively, in which case $\theta_{\mathrm{s}}{ }^{2} \propto s \propto t$. In the unlikely event that $\iota=0$ (the line of sight is directly along the axis; Figure 7), then $\theta_{\mathrm{s}}{ }^{2} \approx$ constant for a narrow outflow. Adopting the jet/cone geometry, the time dependence for the peak flux density is

$$
S_{\nu_{m}}=\theta_{\mathrm{s}}^{2} I_{\nu_{m}} \propto t^{-19 / 18} \approx 1 / t .
$$

Again, from Table 7, the amplitudes of the peaks at time stamps $T_{1}$ to $T_{3}$ are in the ratio, $S_{T_{2}} / S_{T_{1}}=398.5 / 432.7=0.92$ and $S_{T_{3}} / S_{T_{1}}=275.4 / 432.7=0.64$, and the predicted ratios are $(1+56 / 342)^{-1}=0.86$ and $(1+196 / 342)^{-1}=0.64$ (which are in agreement to within $7 \%$ or better).

If we repeat the above, assuming that $B_{\perp} \propto 1 / s$, then the result is $S_{\nu_{m}} \propto t^{1 / 9}$, which would result in an increasing flux density with time, which is clearly not observed. Thus, hereafter, we adopt $B_{\perp} \propto 1 / s^{2}$.

For the sake of completeness in the context of our model, we can also find the time dependence of the flux density, $S_{\nu}$, for any fixed frequency, $\nu$, in the optically thin limit (as opposed to $S_{\nu_{m}}$, because $\nu_{m}$ changes with time as found previously). For this case,

$$
S_{\nu}=\theta_{\mathrm{s}}^{2} I_{\nu}=\theta_{\mathrm{s}}^{2} I_{\nu_{1}} J(z)
$$

where $J(z)$ is given by Equation (7) and $I_{\nu_{1}}$ is related to $I_{\nu_{m}}$ by a simple constant. The time dependence of $\theta_{\mathrm{s}}^{2} I_{\nu_{m}}$ is given by Equation (20) and, in the optically thin limit with $p=2$, $J(z) \propto \nu_{1}^{1 / 2}$. Because $\nu_{1} \propto t^{-11 / 9}$, as already found from Equation (17) with $B_{\perp} \propto 1 / s^{2}$ and $s \propto t$, we find

$$
S_{\nu} \propto t^{-5 / 3} .
$$

Such a dependence might be expected at C-band where the emission is optically thin. Note, however, that we base our analysis on the (exact) polynomial spectral fits to all of the data (fluxes and slopes) in Figure 6, rather than on partial spectral fits to the data as displayed in Figure 9 for $T_{2}$. It is readily seen that the shape of the polynomial curves is not always consistent with the C-band flux density lying in the optically thin region, where the $t^{-5 / 3}$ decline should apply. This is because the value of $z=\nu_{6} / \nu_{1}$ in C-band for $T_{1}$ is found to be only 1.74. Figure 8 then shows that $\alpha_{6}=-0.224$. For $T_{2}, z=2.1$ and thus $\alpha_{6}=-0.341$. That is, with increasing time, the spectrum becomes more optically thin, which is consistent with what might be expected from adiabatic expansion, but the fully optically thin regime is later than $T_{2}$.

Although the $T_{2}$ polynomial spectrum is still not convincingly optically thin, it is the best point from which to try the $t^{-5 / 3}$ extrapolation that was carried out for the C-band curve in Figure 5 (dashed curve) as an alternative to the simpler linear extrapolation (dotted curve).

In summary, both the changing frequencies and amplitudes of the radio spectra are in reasonable agreement with a selfabsorbed cone/jet model that is adiabatically expanding and originates at the time of the hard X-ray flare peak.

\subsection{Circular and Linear Polarization}

The most evidently remarkable results concerning polarization are reported in Table 4 for the polarized fluxes and Table 3 for the associated spectral indices. NGC 4845 is in the extension of the Virgo cluster at high Galactic latitude $\left(b=+74^{\circ}\right)$, so these measurements are unlikely to be contaminated by the Milky Way.

Table 4 reports significant values (between $2 \%$ and $3 \%$ ) of circular polarization (circularly polarized fraction, or $\mathrm{cpf}$ ) in L-band at all resolutions. These are large compared with maximum values previously observed in GPS sources $(\approx 1 \%$; 
e.g., O'Sullivan et al. 2013), although not highly discordant, given the uncertainties (Section 3.2). Equally remarkable is the non-detection of circular polarization at C-band. There is evidently an extremely rapid variation of circular polarization with frequency, as reported in Table 3 (i.e., the in-band spectral index of the circularly polarized flux lies between -2.2 and -3.4). This behavior is coupled with no reliable detection of linear polarization. We need to constrain the source structure to fit these facts.

A satisfactory explanation of our observed polarization behavior is difficult to find in the literature. In this section, we investigate several approaches, and then discuss our proposed explanation, which involves conversion from linear polarization to circular polarization via generalized Faraday rotation (see also O'Sullivan et al. 2013).

The intrinsic circular polarization percentage of optically thin synchrotron radiation is $\cot \phi / \gamma_{e}$, where $\phi$ is the pitch angle and $\gamma_{e}$ is the electron Lorentz factor corresponding to the peak frequency. For a $0.01 \mathrm{G}$ field, $\gamma_{e} \approx 200$ in L-band (Equation (78)), or $\gamma_{e} \approx 100$ for a $0.04 \mathrm{G}$ field. Then one would need $\phi=14^{\circ} \rightarrow 26^{\circ}$ (for $\gamma_{e} \approx 200 \rightarrow 100$, respectively) in the observer's frame to account for a $2 \%$ circular polarization percentage. Such a pitch angle reduces the emissivity to about $12 \% \rightarrow 30 \%$ of the maximum $\left(\propto(B \sin \phi)^{3 / 2}\right)$, which, to maintain the observed flux at the same frequency, would require a rather large magnetic field $\left(10^{-1} \mathrm{G}\right)$ and hence magnetic energy $\left(\sim 10^{49} \mathrm{erg}\right)$.

The major difficulty with this explanation, however, is that the intrinsic polarization predicts a spectral index $\left(\overline{\alpha_{V}}\right)$ for the circularly polarized flux density of -0.5 . This conflicts with what we (Table 3) and others (see discussion in O'Sullivan et al. 2013) have observed. A second difficulty is that we detect little or no linear polarization. This would not be expected for optically thin, homogeneous emission. In any case we have already made a case that our frequency observations embrace the transition to self-absorption (Section 4.2.1). The optically thick regime is also necessary for our proposed explanation by generalized Faraday rotation (e.g., Pacholczyk 1973; Jones 1988; Kennett \& Melrose 1998; Beckert \& Falcke 2002; Macquart 2002; Zheleznyakov \& Koryagin 2002; O'Sullivan et al. 2013).

Jones (1988) gives detailed polarization calculations for a generalization of a definite jet model (see Blandford \& Königl 1979), and finds circular polarization levels in the simulations comparable to our own. Unfortunately the calculated linear polarization tends to be of the same order, and Jones does not discuss the frequency dependence in the optical depth transition zone explicitly. His analytic discussion focuses on the surface where the optical depth is one, whereas we suspect the essential behavior to be in transition across this value. The results are complicated by the assumption of inhomogeneity composed of turbulent cells, which is a structure also invoked by Beckert \& Falcke (2002).

Pacholczyk (1973) discusses the conversion of linearly to circularly polarized flux in a source composed of mainly cold electrons with an admixture of relativistic particles. However $\bar{\alpha}_{V}=-1$ in this limit. Linear polarization is suppressed only by the average effect of the turbulent cells, if the field is indeed randomly distributed and the Faraday rotation per cell is large. When the Faraday rotation per cell is small, the circular polarization has the $\nu^{-3}$ dependence near optical depth unity, but the linear polarization is larger (Beckert \& Falcke 2002).
This may have to be depolarized in an inhomogeneous, Faraday rotating screen.

\subsubsection{Generalized Faraday Rotation}

Generalized Faraday rotation occurs (e.g., Kennett \& Melrose 1998) when the orthogonal normal modes in the source plasma are elliptically polarized rather than circularly polarized (effectively linearly polarized), as is the case for standard Faraday rotation. The latter process occurs in a cold (thermal) electron plasma and is proportional to $B_{\|}$. The former process requires an admixture (becoming dominant in a relativistic limit) of relativistic electrons and is proportional to $B_{\perp}^{2}$. Normally, the generalized process produces a cyclic generation of circularly polarized flux from linearly polarized flux (lpf) (this is the "conversion"). That is the case, provided that the plane of linear polarization rotates along the line of sight, so that there is appropriate rotation on the Poincaré sphere (Kennett \& Melrose 1998). Thus, either the magnetic field rotates or some cold plasma must be present to Faraday rotate the plane of polarization. The cyclic variation modulates the frequency dependence of the cumulative phase shift between the two modes. If the wave number difference is $\Delta k$ then this phase shift is $\Delta k s$, where $s$ is the line of sight distance through the source.

It is important to note that the steep in-band $V$ spectral indices of Table 3 apply over a relatively narrow frequency range $(500 \mathrm{MHz})$ where the transition to self-absorption is occurring, as is indicated by the spectral index $\alpha \approx 1$. In a region where relativistic particles dominate (Kennett \& Melrose 1998) and optical depth effects are neglected, the percentage cpf, $V / S$, is proportional to $\Delta k \propto \nu^{-3}$, which is roughly consistent with our observations. As the relativistic particles become less important relative to the cold electrons (a criterion is given in Kennett \& Melrose 1998), the variation tends to $\nu^{-1}$ (Pacholczyk 1973).

We can consider the dominance criterion for relativistic particles (denoted with subscript, er) over thermal ("cold") particles (denoted, ec). This criterion is $n_{\mathrm{ec}} \ll \gamma_{1} n_{\mathrm{er}} \ln$ $\left(2 \pi \nu / \nu_{\mathrm{g}} B \gamma_{o}\right)$ (recall $\nu_{\mathrm{g}}$ is the gyrofrequency; Equation (5)), which requires $n_{\mathrm{ec}} \ll 2.3 \times 10^{2} \mathrm{~cm}^{-3}$ if $B=0.01$ and $\gamma_{o} \approx 5$ (e.g., Beckert \& Falcke 2002) at L-band. This is about the same order of magnitude as the density of relativistic particles estimated in Section 4.2.1 and implies that the thermal mass in the source is probably of the same order of magnitude as the relativistic particle mass. We pursue this further in Appendix E.

The only similar result of which we are aware is for the quasar PKS B2126-158 as reported in O'Sullivan et al. (2013). They find $\overline{\alpha_{V}}$ to be $-3 \pm 0.4$ at somewhat higher frequency $(8 \mathrm{GHz})$ compared with our result. Their brightness spectrum peaks at a frequency similar to what we infer in Figure 6. Moreover, at lower frequencies, where $I_{\nu} \propto \nu$ as in our spectrum, they detect rising circular polarization between $0.5 \%$ and $1 \%$ in what is the optically thick region. This quasar source thus displays a slightly broader transition between SSA and an optically thin regime. Significantly, when compared to our results, the observed linear polarization is also nearly zero near the peak of the spectrum. This is where their observed circular polarization is largest, much as in our case. These authors explain their $-3 \pm 0.4$ spectral index in circular polarization in the optically thin regime via linear to circular conversion (Kennett \& Melrose 1998; see next subsection), 
which we also adopt in part, although our measurements are taken closer to the optically thick transition.

\subsubsection{Conversion of Linear to Circular Polarization}

The mechanism described previously, of course, requires an initial linear polarization to be rotated on the Poincaré sphere. Fortunately, Beckert \& Falcke (2002) give a relatively simple set of equations together with a complete set of absorption and emission coefficients for a relativistic plasma, which allow us to investigate the spectral dependence and conversion from linear to circular polarization in more detail. We develop these in Appendix E and extend their analysis to include the case $p=2$, as implied by our total intensity spectrum (Section 4.2.1).

We consider the two limiting cases of small and large Faraday depth. For the core of NGC 4845, we also show numerically that such conversion can explain the circularly polarized flux that is observed. However, the true condition in the source is likely to be intermediate between these limits. The low Faraday rotation source plasma is the limit closer to what we observe. The circularly polarized flux is given by Equation (67) as a blend of synchrotron emissivity and rotational conversion, which agrees with our observed $\overline{\alpha_{V}}$. Should $B(-2)$ be as large as 10 , the required linear polarization for conversion is reduced to typical values (3\% or $4 \%$ ). To reduce the linear polarization further, however (i.e., to less than $0.5 \%$ as observed), it must still be suppressed by an inhomogeneous, depolarizing medium. The presence of such a medium is not unusual for edge-on galaxies; a comparison to other galaxies in the CHANG-ES sample (Wiegert et al. 2015) shows that a large number also lack lpf at the L-band.

We conclude that to explain our observations we need only that the relativistic particles are present and are dominant at L-band. The boundary conditions $U=U_{o}$ and $Q=V=0$ do suggest that we are observing an organized synchrotron source through an inhomogeneous medium of mixed cold and hot particles (e.g., Zheleznyakov \& Koryagin 2002). For a coherent synchrotron source near the jet axis (see Appendix A for the general scheme), we may suppose that our calculations apply to a sheath where relativistic particles still dominate. The depolarizing screen around the jet possessing dominant Faraday rotation may gradually appear due to an influx of entrained thermal particles. The circular polarization incident on the screen should not be affected by the Faraday rotation in the screen. This picture is similar to a two jet model (Wang et al. 2014), except that the outer jet here contains a mixture of relativistic and thermal electrons. The thermal particles presumably become dominant at larger scales, perhaps due to entrainment of a surrounding thermal cloud.

\subsection{A Proposed Radio/X-Ray Connection}

\subsubsection{Inverse Compton Radiation}

We first consider whether inverse Compton radiation (the socalled synchrotron self-Compton, or SSC mechanism, for upscattering from radio to $\mathrm{X}$-ray energies) can explain the $\mathrm{X}$-ray emission, given the properties as measured in the radio source now.

Inverse Compton emission is related to synchrotron emission from an ensemble of relativistic particles through the ratio of the radiation energy density to the magnetic energy density, $U_{\mathrm{rad}} / U_{\mathrm{mag}}$, that is, the SSC flux would be $S_{\mathrm{SSC}}=S_{\mathrm{R}} U_{\mathrm{rad}} /$
$U_{\mathrm{mag}}$, where $S_{\mathrm{R}}\left(\mathrm{erg} \mathrm{s}^{-1} \mathrm{~cm}^{-2}\right)$ is the measured radio flux. We estimate the radiation energy density from $U_{\mathrm{rad}}=(4 \pi / c) I$, so that

$$
U_{\mathrm{rad}} \approx \frac{16}{c} \frac{\nu S_{\nu}}{\theta_{\mathrm{s}}^{2}} \approx 5 \times 10^{-7} \frac{S_{(4.87)} \nu_{(4.87)}}{\theta_{\mathrm{s}}(\mathrm{mas})^{2}} \mathrm{erg} \mathrm{cm}^{-3}
$$

We have taken the maximum from the earliest epoch in Figure 6 when the frequency is $4.87 \mathrm{GHz}$ (letting $\nu_{(4.87)}=1$ ) and the flux density at that frequency is $433 \mathrm{mJy}$ (Table 7), or $S_{(4.87)}=1$, in Equation 23. Using $U_{\mathrm{mag}}=B^{2} /(8 \pi)$, we find the ratio

$$
\frac{U_{\mathrm{rad}}}{U_{\mathrm{mag}}} \approx 0.12 \frac{S_{(4.87)} \nu_{(4.87)}}{\theta_{\mathrm{s}}(\mathrm{mas})^{2} B(-2)^{2}} .
$$

We might therefore expect inverse Compton emission during the radio outburst at about $10 \%$ of the radio flux density using $B(-2)=1(0.01 \mathrm{G})$ for the field strength (Appendix F). This emission should be in a band about the peak value $\gamma_{e}^{2} \nu_{\text {peak }}$. Using our previous estimate for $\gamma_{e}$ (estimated for L-band; Section 4.4), we expect this to lie in the near-infrared to optical bands-not the X-ray.

To limit any X-ray flux, we can find the maximum radio flux from our observations by integrating over the band from 0.5 to $10 \mathrm{GHz}$ for the highest curve of Figure 6, finding $\approx 2 \times 10^{-14} \mathrm{erg} \mathrm{s}^{-1} \mathrm{~cm}^{-2}$. The X-ray light curve in the $17.3-80 \mathrm{keV}$ band ranges from $10^{-13} \rightarrow 8 \times 10^{-11} \mathrm{erg} \mathrm{s}^{-1} \mathrm{~cm}^{-2}$ (Nikolajuk \& Walter 2013, their Figure 8).

The last point, in particular, in Figure 8 of Nikolajuk \& Walter (2013) overlaps in time with our observations. Whatever the origin of this X-ray emission is, it cannot be due to SSC from the radio. Not only is the luminosity low, but the Compton scattered frequency of the radio photons falls far below the X-ray band. Thus, another source of X-ray emission is independent of the SSC mechanism. In Nikolajuk \& Walter (2013) it is recognized that this last point does not fit the expected tidal disruption behavior that is found near the peak $\mathrm{X}$-ray emission. It is likely that some late behavior arising near the accreting disc of the system (which is perhaps at $\sim 1 \mathrm{AU}$ from the black hole) is responsible.

\subsubsection{Inverse Compton Radiation from an Evolving Source}

If the radio emission does not explain the $\mathrm{X}$-ray emission via SSC for current conditions, can we explain the X-ray emission by extrapolating the radio conditions to the epoch of the peak $\mathrm{X}$-ray emission?

A remarkably similar set of observations to our own were reported for the radio emission associated with a Swift Gammaray source (Zauderer et al. 2011). These authors concluded that they were seeing the birth of a parsec scale radio jet in the core of a rather distant galaxy. Like us (previous subsection), these authors rejected inverse Compton emission because of the luminosity shortfall, as well as the lack of correlation between the radio and X-ray variability.

However, in our case, the X-ray energy distribution power law near the peak emission $\left(p_{\mathrm{X}} \approx 2.2\right.$; Nikolajuk \& Walter 2013) is similar to the radio energy distribution power law $(\approx 2$; Section 4.2.1), and both X-ray and radio emission consist of a single, strong point-like source at the center of the galaxy. We are thus further motivated to attempt to establish a potential link in the jet development. A complete description of our 
development is given in Appendix F and is within the context of the jet model of Appendix A. We summarize the arguments and results here.

Recall that timescale arguments (Section 4.1) suggest a radio source size of order $0.1 \mathrm{pc}(\sim 1 \mathrm{mas})$ and, if the jet is relativistic (Appendix A), we expect that the shocked X-ray emitting region at the earlier time (the epoch of the peak X-ray emission about one year earlier) is an order of magnitude smaller in size; a region that we refer to as the X-ray "lobe." The observed flux density of the circular polarization is consistent with a magnetic field strength of order $10^{-2} \mathrm{G}$ (these are order of magnitude estimates). Along with the observed flux density of the peak of the radio emission at time $T_{1}$, the inputs constrained by observation are the only inputs to our model.

The extrapolation allows the magnetic field strength in the $\mathrm{X}$-ray lobe to be estimated because the variation with size is known for a constant velocity jet $\left(B \propto 1 / r^{2}\right.$; Appendix A). Since the X-ray frequency is known, an estimate of the Lorentz factor that is required to produce the X-rays via SSC can be determined, along with the critical frequency of synchrotron photons in the X-ray source. We find that the synchrotron emission in the X-ray lobe would peak in the infrared (IR).

The flux of the IR synchrotron radiation in the X-ray lobe can be found by relating the currently observed synchrotron emission of the radio source to the IR synchrotron emission in the X-ray lobe, knowing how synchrotron emission depends on the Lorentz factor, the magnetic field, the frequency, the source size, the electron energy power law $(p=2)$, and the electron particle constant, $N_{0}$, (which depends on time in a known fashion for an adiabatically expanding source; see Section 4.3). The X-ray flux from SSC, $S_{\mathrm{X}_{\mathrm{SS}}}$, in the X-ray lobe can then be determined from the IR synchrotron emission and magnetic field (see previous subsection).

Our calculations (Appendix F) for these conditions show that $U_{\mathrm{rad}_{\mathrm{IR}}} / U_{\mathrm{mag}}=1.1$ and $S_{\mathrm{X}_{\mathrm{SSC}}}=2.0 \times 10^{-11} \mathrm{erg} \mathrm{s}^{-1} \mathrm{~cm}^{-2}$; the latter value falls short of the peak observed X-ray flux of $S_{\mathrm{X}}=8.0 \times 10^{-11} \mathrm{erg} \mathrm{s}^{-1} \mathrm{~cm}^{-2}$ (Nikolajuk \& Walter 2013) by a factor of four. The result is, however, very sensitive to the magnetic field in the X-ray lobe. We have, so far, allowed for an increase in the magnetic field to the earlier epoch, which only accounts for a change in size via jet geometry, and have not yet allowed for the possibility that shocks in the X-ray lobe may enhance the magnetic field further. If we include a modest enhancement of the magnetic field (a factor of 1.8, whereas a strong shock would give 4), then we find the same ratio of $U_{\mathrm{rad}_{\mathrm{IR}}} / U_{\mathrm{mag}}$ and $S_{\mathrm{X}_{\mathrm{SSC}}}=8 \times 10^{-11} \mathrm{erg} \mathrm{s}^{-1} \mathrm{~cm}^{-2}$, which is in agreement with the X-ray observations.

Given these results, we are encouraged to discuss such an SSC X-ray source in more detail (see Figure 7). We imagine that largely thermal material at a very high temperature is expelled from near the black hole due to the tidal disruption of a Jupiter-sized object. The disrupted material also forms a disc or torus near $1 \mathrm{AU}$. The ejected thermal material is collimated into a fast beam by this disc. Subsequently, the beam collides with a cloud of interstellar material at about $0.01 \mathrm{pc}$ and forms an X-ray-emitting region (the X-ray "lobe"). This lobe consists of a forward shock in the cloud, a reverse shock in the beam, and a separating contact discontinuity in the medium between the shocks. The model is much as was described in another context in Henriksen et al. (1991).

For the shock wave acceleration to be effective in accelerating the electrons, the acceleration time, $t_{\mathrm{acc}}$, must be less than the synchrotron lifetime. In Henriksen et al. (1991) this was estimated in their Equation (7). For relativistic shock speeds (it is sufficient for $v_{\text {shock }}$ to be $>0.001 c$ ) and our magnetic field ( $B$ in the X-ray lobe of $1.8 \mathrm{G}$ ), $t_{\mathrm{acc}}<10 \mathrm{~min}$ (which is proportionately shorter for higher $v_{\text {shock }}$ ). The synchrotron lifetime of an electron (Equation (52)) under these conditions with $\gamma_{e}=1109$ (Appendix F) on the other hand, is about 20 minutes. Hence the acceleration time is sufficiently short.

This result also indicates that there must be continuous injection or acceleration of the electrons in the X-ray lobe over a time during which the SSC mechanism is producing the hard $\mathrm{X}$-ray emission. From the X-ray light curve (Nikolajuk \& Walter 2013), this would occur around the peak of the light curve and up to $\approx 120$ days after the peak if the first cluster of $\mathrm{X}$-ray measurements after the peak is produced in the same way. After that time, the X-ray light curve declines precipitously (suggesting that there is no longer continuous injection/acceleration), revealing a secondary X-ray-emitting source, which is possibly associated with the accretion disk.

If the reverse shock is a very strong shock, it produces an accelerated distribution of electrons with an energy power law, $N_{o} E^{-3 / 2}$ (Malkov \& Drury 2001; i.e., the injection spectrum is described by $p_{\text {inj }}=3 / 2$ ). If this is the continuous injection spectrum for the electrons from this shock, then according to Longair (1994), above an energy given by

$$
E_{\mathrm{s}}=\frac{1.2 \times 10^{7}}{B_{\mathrm{s}}^{2} t_{\mathrm{s}}\left(1+\frac{U_{\mathrm{rad}}}{U_{\mathrm{mag}}}\right)} \mathrm{eV}
$$

(where $t_{\mathrm{s}}$ is the source lifetime in years and $B_{\mathrm{s}}$ is the field in Gauss), the spectrum steepens from synchrotron losses such that $p=p_{\text {inj }}+1=2.5$. The required electrons are well above this energy and therefore we expect a power law, $E^{-2.5}$, from this shock. If the forward shock is less strong, then we expect the test particle result, $p=2$, to be given to the electrons by this shock. The electrons scattered upstream of the forward shock will form the radio spectrum with $p=2$. These must be a significant fraction of the electrons scattering photons to X-ray energies, but not in energy space energies. The accelerated electrons that leave the shock in the forward direction cool rapidly by synchrotron radiation and eventually form the adiabatically expanding radio lobe that we infer from our observations. The cooling time is rapid enough to convert the high energy electrons that leave the X-ray lobe into the low energy radio electrons that are observed about a year after the peak.

In summary, in the context of this model, the regions of the shocks of size $\approx 1000 \mathrm{AU}$ and magnetic field $\approx 1 \mathrm{G}$ can produce X-ray emission via SSC (the X-ray "lobes") with outflow in the forward direction developing into the radio jet. Relativistic electrons cool rapidly after leaving the shocked region, and develop into an adiabatically expanding outflow.

\section{CONCLUSIONS}

Our main conclusions are as follows.

1. The radio emission from the Virgo Cluster spiral, NGC 4845, is dominated by a single unresolved point source that has increased by a factor of $\approx 6$ since the 1995 
NVSS survey and also varied over the $\approx 6$ month timescale of the CHANG-ES observations (Table 4). Prior to NVSS, there is no evidence for radio variability from the few data points that exist (Section 3.1.4). However, some vertical radio extensions as well as a distinct optical cone (Figure 2) suggest that previous outflow has likely occurred.

2. A small central disk of diameter $1.8 \mathrm{kpc}$ surrounds the nucleus, but constitutes only $19 \mathrm{mJy}$ at L-band (less than $10 \%$ of the total flux density) and $7 \mathrm{mJy}$ at C-band (less than $2 \%$ of the total). The spectral index of this disk is $\alpha_{\text {disk }}=-0.74$ (Section 3.1.1).

3. The CHANG-ES observations overlap in time with the hard X-ray light curve of Nikolajuk \& Walter (2013, their Figure 8). The X-ray flare, interpreted as being due to a tidal disruption event (TDE), provides a well-defined reference point for the origin of the current radio emission. We provide a simple jet model for the radio emission (Appendix A and Figure 7), which is relevant whether the X-ray outflow originated with a TDE or is due to infall from an accretion disk, although the former may lead to the latter in any case.

4. Variability suggests a source size of $\theta_{s} \leqslant 4 \sin (\iota)$ mas (of order $0.1 \mathrm{pc}$ ), where $\iota$ is the viewing angle (Section 4.1).

5. The wide bands used in the CHANG-ES survey have allowed us to determine in-band spectral indices and to solve explicitly for the spectrum of the AGN as a function of time (Section 3.1.5 and Figure 6). At L-band $\alpha_{1.57 \mathrm{GHz}} \approx+1.0$ and at $\mathrm{C}$-band, $\alpha_{6.00 \mathrm{GHz}} \approx-0.5$ (Table 3), suggesting an energy spectral index for the relativistic electrons of $p=2.0$ and that NGC 4845 is a nearby example of a "GPS" radio source.

6. The turnover in the spectrum can be explained by SSA (Section 4.2.1). However, a contribution from thermal absorption at L-band is possible.

7. The peak of the radio spectrum both declines and moves to lower frequencies with time (Figure 6). This behavior can be understood in terms of adiabatic expansion (Section 4.3).

8. We detect circular polarization (Section 4.4) of order a few percent in the source, although no significant linear polarization, at L-band (Table 4). The circularly polarized flux has a steep spectral index $(\approx-3$, consistent with other authors) and can be explained by conversion from linear polarization - a natural consequence of generalized Faraday rotation for a source that is dominated by relativistic particles (see Beckert \& Falcke 2002, and Appendix E). A further reduction of lpf requires an additional foreground depolarizing screen.

9. Inverse Compton emission from the currently observed radio source is insufficient to account for the X-ray emission detected by Nikolajuk \& Walter (2013).

10. However, if we extrapolate to the peak of the X-ray light curve at which time the source size is smaller and the magnetic field higher (Figure 7), the X-ray emission can be explained by inverse Compton upscattering by the relativistic particles (SSC), which is a result supported on energetic grounds and also by the similarity between spectral indices at radio and X-ray wavelengths (Section 4.5.2).

NGC 4845 appears to be a young nuclear radio jet/outflow and is, by far, the closest known GPS source. As such, it provides a unique opportunity to study an evolving radio source associated with a measured hard X-ray burst. The radio source, after five years at an assumed bulk outflow speed of $c / \sqrt{3}$ (Appendix A), would have expanded to about $1 \mathrm{pc}$, or 12 mas. This size is within the realm of VLBI. With observations over several years, it may be possible to directly observe the proper motion and evolution of this source and further piece together its connection to hard X-ray outflows.

This work has been supported by a Discovery Grant to the first author by the Natural Sciences and Engineering Research Council of Canada. This research has made use of the NASA/ IPAC Extragalactic Database (NED) which is operated by the Jet Propulsion Laboratory, California Institute of Technology, under contract with the National Aeronautics and Space Administration. The National Radio Astronomy Observatory is a facility of the National Science Foundation operated under cooperative agreement by Associated Universities, Inc.

Facilities: VLA.

\section{APPENDIX A}

\section{THE STANDARD JET MODEL}

We are imagining a shocked section of a conical outflow (or possibly the pseudo core discovered in Jones 1988) as the source region. Figure 7 shows the geometry.

The jet model summarized here has been assumed as the synchrotron source in AGN by many authors, beginning with Blandford \& Königl (1979) and then Jones (1988). However, the dynamical details of such sources are not readily found in the literature. Moreover, we are able to suggest a novel observational idea based on the net current flow through the jet. For these reasons we have decided to include a description of the model in this appendix. Ultimately it will form the basis of an analysis in the style of Jones (1988), which is the level required to match more abundant observations. The simple model described here is similar to that used by Jones (1988), however we treat the magnetic field in more detail and include a decelerating branch of the outflow. Whether this branch or the more familiar accelerating branch appears depends on ambient boundary conditions. In addition, we consider the possibility of change in the direction of the current flow in the jet and corresponding change in the magnetic field.

One assumes that the jet originates near the compact object as a gas dominated by relativistically hot particles. Moreover, if $p$ is the gas pressure and $\rho$ is the total energy density (including the thermal energy), ${ }^{15}$ then we assume for simplicity the "polytropic" relation (we take units with $c=1$; e.g., $a_{\mathrm{s}} c$ is the sound speed in usual units)

$$
p=a_{\mathrm{s}}^{2} \rho .
$$

One defines the conserved rest mass density $\mu$ according to $p+\rho=\mu(d \rho / d \mu)$, so that after inserting the pressure and integrating,

$$
\frac{\rho}{\rho_{\mathrm{s}}}=\left(\frac{\mu}{\mu_{\mathrm{s}}}\right)^{1+a_{\mathrm{s}}^{2}} .
$$

\footnotetext{
15 We retain "standard" nomenclature that is consistent within this appendix but may differ from the main text (e.g., $p$ refers to pressure here, whereas it was used as the energy spectral index in the text; likewise for subsequent appendices).
} 
The constants with subscripts, s, are evaluated at some convenient reference point.

It is also useful, when expressing the dynamical equations, to define the internal energy per unit rest mass energy as

$$
\xi \equiv \frac{p+\rho}{\mu}=\left(1+a_{\mathrm{s}}^{2}\right) \frac{\rho_{\mathrm{s}}}{\mu_{\mathrm{s}}}\left(\frac{\mu}{\mu_{\mathrm{s}}}\right)^{a_{\mathrm{s}}^{2}} .
$$

Hence also

$$
\rho=\frac{\xi \mu}{1+a_{\mathrm{s}}^{2}} .
$$

We assume strictly radial flow, $u$, in the jet, together with a steady state. This renders the transverse equilibrium equation useless because it only defines some unknown confining force in the theta direction. We are left with energy conservation along the flow direction together with a rest energy conservation law. When written in the observer frame of reference these yield, respectively, after a slight rearrangement,

$$
\xi \gamma(u)=\mathcal{E},
$$

and

$$
r^{2} \mu \gamma(u) u=A .
$$

Here $\mathcal{E}$ is essentially the constant specific energy in the jet and $A$ is the constant rest "mass" flux through the jet.

For the energy conservation to take this simple form, there can be no Ohmic dissipation or other working on the jet by the electromagnetic field. Assuming that the electric field vanishes in the comoving frame $(\boldsymbol{E}=-\boldsymbol{u} \wedge \boldsymbol{B})$ and that the magnetic field in the observer frame is $\boldsymbol{B}=B_{r} \widehat{\boldsymbol{e}}_{r}+B_{\phi} \widehat{\boldsymbol{e}}_{\phi}$, then $\boldsymbol{E}=u \boldsymbol{B}_{\phi} \widehat{\boldsymbol{e}}_{\theta}$; and so we require the poloidal current density $j_{\theta}=0$ in order for the dissipation $\boldsymbol{j} \cdot \boldsymbol{E}=0$. In fact, one expects to have $\boldsymbol{j} \| \boldsymbol{B}$, although this is not consistent with purely radially moving charges. It can only be the bulk flow that moves wholly radially.

The last two equations in this simplified form suffice to determine the jet dynamics as a function of $r$, as we will report below. However, the question as to the evolution of the magnetic field arises.

The rest mass conservation law and Faraday's law can be combined to give the equation satisfied by the advected magnetic field on each stream line in the form

$$
\frac{d}{d t}\left(\frac{\boldsymbol{B}}{\mu \gamma(u)}\right)=\left(\frac{\boldsymbol{B}}{\mu \gamma(u)} \cdot \nabla\right) \boldsymbol{u},
$$

which for purely radial velocity integrates to give

$$
\begin{aligned}
\frac{B_{r}}{\mu \gamma(u)} & =\left(\frac{B_{r}}{\mu \gamma}\right)_{\mathrm{s}} \frac{u(r)}{u_{\mathrm{s}}} \\
\frac{B_{\phi}}{\mu \gamma(u)} & =\left(\frac{B_{\phi}}{\mu \gamma}\right)_{\mathrm{s}} \frac{r}{r_{\mathrm{s}}} .
\end{aligned}
$$

Magnetic flux conservation is contained in these equations, provided that $\nabla \cdot \boldsymbol{B}_{\mathrm{s}}=0$.

To understand the significance of $B_{\phi s}$ we consider the radial component of the current. Restoring $c$ for the moment and

\footnotetext{
16 "Radius" refers to the radial direction; the cross-sectional radius of the cone is referred to as $r \sin \theta$.
}

taking account of the axial symmetry one finds at any radius ${ }^{16}$

$$
B_{\phi}=\frac{2}{c r} \frac{I_{r}(\theta)}{\sin \theta},
$$

where $I_{r}(\theta)$ is the total current inside the angle $\theta$. For a zero net current through the central source, counting both the jet (polar angle $\theta_{j}$ ) and a likely sheath (polar angle $\theta_{\mathrm{sh}}>\theta_{j}$ ), we must have $I_{r}\left(\theta_{\mathrm{sh}}\right)=0$. The only way in which the azimuthal magnetic field can reverse sign however is if there is a net current toward or away from the central source.

For example, let us assume that the radial current density through the jet is of the form

$$
j_{r}=\frac{I}{r^{2}}\left(\tan \theta_{+}-\tan n \theta\right) \sin \theta,
$$

where $\tan \theta_{+}$is a convenient parameter and $n$ is an integer that determines how narrow the jet is. Then the radial current is found to be $(n=2)$

$$
\begin{aligned}
I_{r}= & 2 \pi I\left(\tan \theta_{+}\left(\frac{\theta}{2}-\frac{\sin 2 \theta}{4}\right)+\frac{\ln (\cos 2 \theta)}{4}\right. \\
& \left.+\frac{1}{4}(1-\cos 2 \theta)\right) .
\end{aligned}
$$

When $\theta_{+}=\pi / 6$ and $n=2$ the current passes through zero at $\theta=19^{\circ} .7$. Beyond this angle the net current is negative. A balancing current must flow in the more diffuse surroundings or on the surface in order to prevent the accumulation of a central charge. Such shearing magnetic fields (e.g., Equation (34)) can lead to circular polarization (e.g., Zheleznyakov \& Koryagin 2002) and possible rapid variations in the observed rotation measure (G. Heald 2015, private communication).

The interaction of the radial current density and the azimuthal magnetic field leads to a "pinching" or collimating force. The parameters might be chosen so as to balance the transverse equation at the reference point or "nozzle." However the assumed conical geometry is not generally consistent with the subsequent transverse force equation, so we do not attempt this here.

The model does not yet allow for the acceleration of the jet by the magnetic field. The Poynting flux in the radial direction due to the wound-up "spring" component of the field at any point, $r_{\mathrm{s}}$, is $u\left(r_{\mathrm{s}}\right) B_{\phi s}^{2} / 4 \pi$. The associated energy density is then $B_{\phi s}^{2} / 4 \pi$. One might try to include this effect by adding the quantity $a_{a}^{2} \equiv B_{\phi s}^{2} /\left(4 \pi \rho_{s} c^{2}\right)$ to $a_{\mathrm{s}}^{2}$ in the expression for pressure. However we find that this quantity is not constant in radius, so the approximation can only apply over a small section of the jet compared to its length. Hereafter we will also ignore this possibility, but it can be restored locally simply by modifying $a_{\mathrm{s}}^{2}$ to $a_{\mathrm{s}}^{2}+a_{a}^{2}$.

The jet velocity profile follows from Equations (30) and (31), once $\mu$ is eliminated in favor of $\xi$ and hence $\gamma$. We can eliminate most of the parameters by writing $x=r / R$, where (after restoring $c$ )

$$
R^{2} \equiv \frac{A}{\mu_{\mathrm{s}} c}\left(\frac{\rho_{\mathrm{s}}\left(1+a_{\mathrm{s}}^{2}\right)}{\mu_{\mathrm{s}} \mathcal{E}}\right)^{1 / a_{\mathrm{s}}^{2}} .
$$


The velocity in the jet as a function of scaled radius in units of $c$ is then found from

$$
x^{2}=\frac{1}{\beta\left(1-\beta^{2}\right)^{\frac{1-a_{\mathrm{s}}^{2}}{2 a_{\mathrm{s}}^{2}}}} .
$$

This expression gives the velocity at $x$ for any particular value of $a_{\mathrm{s}}^{2}$, although it contains some surprises. As $x \rightarrow \infty$ one can have two branches for $a_{\mathrm{s}}^{2}<1$. In the accelerated branch $\beta \rightarrow 1$, whereas in the decelerated branch $\beta \rightarrow 0$. The choice is made by the boundary conditions at infinity, which apply a finite pressure in the decelerated jet flow and vanishing pressure in the accelerated jet flow.

It is also clear that there is a minimum radius at which these two branches can exist. If we calculate $d x^{2} / d \beta=0$ we find that at this minmum, which we may take to be $x_{\mathrm{s}}$,

$$
\begin{aligned}
\beta & =a_{\mathrm{s}}, \\
x_{\mathrm{s}}^{2} & =\frac{1}{a_{\mathrm{s}}\left(1-a_{\mathrm{s}}^{2}\right)^{\frac{1-a_{\mathrm{s}}^{2}}{2 a_{\mathrm{s}}^{2}}}} .
\end{aligned}
$$

The minimum point thus represents the origin of the jet in a "sonic" launch point. The two branches meet at this point and there is no flow extending to smaller radius with $\beta>0$. This behavior is due to the absence of a restraining force such as gravity, which, if present would turn this minimum point into a true choke point in the jet flow or wind. Once Equation (38) is solved, we find $\xi$ from Equation (30) and hence $\mu, \rho, B_{r}$, and $B_{\phi}$ from the various relations.

We can be more explicit in the interesting case where $a_{\mathrm{s}}^{2}=1 / 3$ (i.e., the jet velocity is the sound speed expected for relativistic internal energy, $u=c / \sqrt{3}$, when $c$ is restored). Equation (38) becomes the parameter-free cubic equation

$$
\beta^{3}-\beta+\frac{1}{x^{2}}=0,
$$

and $x_{\mathrm{s}}^{2}=\sqrt{27} / 2$ and $\beta_{\mathrm{s}}=1 / \sqrt{3}$. The discriminant of this equation is $\Delta=-4+27 / x^{4} \equiv 4\left(-1+x_{\mathrm{s}}^{4} / x^{4}\right)$. When $\Delta>0$ so that $x<x_{\mathrm{s}}$ there is only one real root and it is negative. At $x=x_{\mathrm{s}}$ we have $\Delta=0$, and there is only one real (double) root $\beta_{\mathrm{s}}=1 / \sqrt{3}$.

In the region of interest, $x>x_{\mathrm{s}}$, we have $\Delta<0$ and there are three real roots but only two are positive. The positive roots take a convenient form: for the accelerated branch as

$$
\beta_{\mathrm{acc}}=\frac{2}{\sqrt{3}} \cos \left(\frac{1}{3} \arccos \left(-\frac{\sqrt{27}}{2 x^{2}}\right)\right),
$$

and for the decelerated branch as

$$
\beta_{\mathrm{dec}}=\frac{2}{\sqrt{3}} \cos \left(\frac{4 \pi}{3}+\frac{1}{3} \arccos \left(-\frac{\sqrt{27}}{2 x^{2}}\right)\right) .
$$

These give, respectively, $\beta_{\mathrm{acc}} \rightarrow 1$ and $\beta_{\mathrm{dec}} \rightarrow 0$ as $x \rightarrow \infty$. At $x=x_{\mathrm{s}}$ each expression yields the root $\beta_{\mathrm{s}}=1 / \sqrt{3}$ as expected. The general behavior is illustrated in Figure 10. The curves actually meet vertically at $x_{\mathrm{s}}$.

The behavior at large $x$ is easy to determine using Equations (41) and (42). For the accelerated jet one finds in

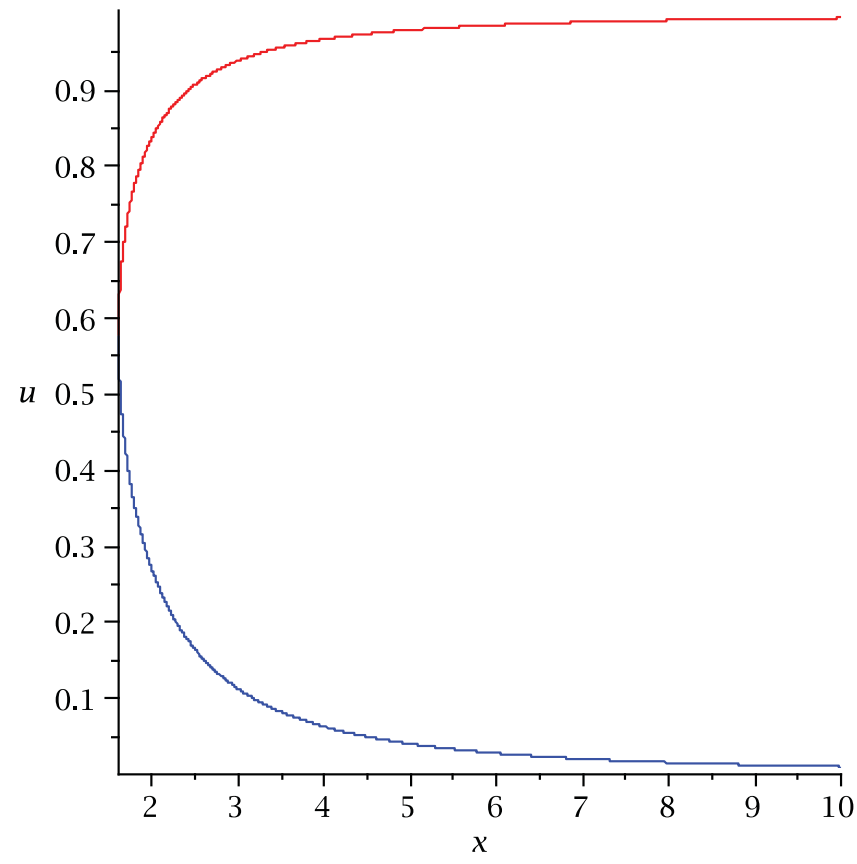

Figure 10. Upper curve is the accelerated jet universal velocity behavior when $a_{\mathrm{s}}^{2}=1 / 3$ in terms of $x=r / R$. The lower curve is the decelerated jet velocity under the same conditions. The pressure at infinity goes to zero to establish the upper curve, whereas it retains a finite value on the lower curve. The curves meet vertically at the central value of $u=1 / \sqrt{3}=0.57735$.

lowest order $(\asymp$ means equals in the asymptotic limit)

$$
\begin{aligned}
& \beta \asymp 1-\frac{1}{2 x^{2}}, \\
& \gamma \asymp x, \\
& \xi \asymp \frac{\mathcal{E}}{x}, \\
& \mu=\left(\frac{3 \mu_{\mathrm{s}}}{4 \rho_{\mathrm{s}}}\right)^{3} \xi^{3}, \\
& \rho=\frac{3}{4} \xi \mu .
\end{aligned}
$$

Equations (33) then show that, asymptotically, $B_{r} \propto x^{-2}$ and $B_{\phi} \propto x^{-1}$ as is normally assumed. The behavior of the magnetic field near the source is however more complicated. This is the branch describing the jet expanding into a low pressure ambient medium.

For the decelerated jet, the behavior follows from Equation (42) as

$$
\begin{aligned}
& \beta \asymp \frac{1}{x^{2}}, \\
& \gamma \asymp \frac{1}{\sqrt{1-1 / x^{4}}}, \\
& \xi=\mathcal{E} \sqrt{1-1 / x^{4}},
\end{aligned}
$$

and the expressions for $\mu$ and $\rho$ follow from the dependences on $\xi$ given in Equations (43). Boundary conditions at infinity are determined by condtions at the origin $x_{\mathrm{s}}$ and vice versa. The radial field varies again as $x^{-2}$ in the lowest order, but the azimuthal field increases as $B_{\phi} \propto\left(x-1 / x^{3}\right)$. Even in the absence of shocks, this can lead to an outer brightening of the 
jet. However, a dense medium at infinity such that $\xi_{\infty}=\mathcal{E}$ is required to decelerate the jet. This dense medium may be composed of material swept-up from the ambient medium.

\section{APPENDIX B \\ SOURCE PROPER FRAME PROPERTIES}

We have used quantities in the local rest frame for our calculations. This ignores the possible relativistic motion of the source. In the presence of rest frame gas against which the source is expanding, a deceleration to non-relativistic source velocity is possible (see, e.g., Appendix A). However it is important to be aware of the modifications required by relativistic source motion. We discuss these in this section. Since NGC 4845 is not at large cosmological redshift, we do not include this effect.

In the first instance we argued generally in terms of the standard isotropic, energy power law distribution of source electrons (or possibly positrons). However, a particle moving in the source frame at an angle $\theta^{\prime}$ to the relative velocity appears at an angle $\theta$ to the velocity in the local rest frame, where $\theta$ is (e.g., Henriksen 2011)

$$
\tan \theta=\frac{\sin \theta^{\prime}}{\gamma_{u}(u)\left(\cos \theta^{\prime}+u / v^{\prime}\right)},
$$

where $u$ is the relative velocity, $v^{\prime}$ is the source particle speed, and $\gamma_{u}$ is the bulk flow Lorentz factor. The inverse relation is found by interchanging the primes and replacing $u$ with $-u$.

This transformation shows that only with $u / v^{\prime} \ll 1$ and $\gamma_{u} \approx 1$ do the angles coincide and hence isotropy is invariant. Otherwise, even a source particle moving at $\theta^{\prime}=90^{\circ}$ when $\gamma_{u}=3(\beta \approx 0.94)$ and $u / v \approx 1$ is directed at $\theta \approx 18.4$ in the local rest frame. This cone contains all other forward moving particles. Particles with $\theta^{\prime}>90^{\circ}$ are also beamed to a positive angle in the rest frame.

Consequently, an isotropic distribution of particles in the source frame is a directed beam in the observer frame. Fortunately the beaming due to synchrotron radiation itself tends to dominate this effect in the integration over the solid angle.

The energy of the particles transforms according to

$$
E=\gamma_{u}\left(1+\frac{\boldsymbol{u} \cdot \boldsymbol{v}^{\prime}}{c^{2}}\right) E^{\prime}, \quad \text { or } \quad \gamma=\gamma_{u}\left(1+\frac{\boldsymbol{u} \cdot \boldsymbol{v}^{\prime}}{c^{2}}\right) \gamma^{\prime},
$$

where $v^{\prime}$ is the source particle velocity. The inverse follows by interchanging the primes and reversing the sign of $\boldsymbol{u}$. We see that the energy of forwardly moving particles is increased by the roughly constant factor $2 \gamma_{u}$. This implies that the power law distribution in energy in the source frame changes only its limits in the rest frame.

It is of interest to clarify the transformation of intensity or "brightness," $I_{\nu}$. This was originally discussed in this context in Blandford \& Königl (1979), but it is worth repeating the details.

The radiation pattern of a source transforms as (e.g., Henriksen 2011)

$$
\frac{d^{2} E}{d \Omega d t}=\frac{1}{\gamma_{u}^{4} \kappa^{3}} \frac{d^{2} E^{\prime}}{d \Omega^{\prime} d t^{\prime}}
$$

where $\kappa \equiv\left(1-\widehat{\boldsymbol{k}} \cdot \frac{\boldsymbol{u}}{c}\right)$, and $\Omega$ denotes a solid angle. The unit vector $\widehat{\boldsymbol{k}}$ lies along the direction of a ray. For a distant observer, however, the time is $t_{\mathrm{obs}}=t+R / c$ and hence $d t_{\mathrm{obs}}=d t \kappa$, where $\mathrm{R}$ is the distance to the observer along the emitted ray. Consequently, we have

$$
\frac{d^{2} E}{d \Omega d t_{\mathrm{obs}}}=\frac{1}{\gamma_{u}^{4} \kappa^{4}} \frac{d^{2} E^{\prime}}{d \Omega^{\prime} d t^{\prime}}
$$

However, to calculate the relation between the emissions per unit frequency, we must use the Döppler shift relation $d \nu=d \nu^{\prime} /(\gamma \kappa)$ to obtain

$$
\frac{d^{2} E}{d \Omega d t_{\mathrm{obs}} d \nu}=\frac{1}{\gamma_{u}^{3} \kappa^{3}} \frac{d^{2} E^{\prime}}{d \Omega^{\prime} d t^{\prime} d \nu^{\prime}} .
$$

Finally, to obtain the transformation of the intensity, we must consider an area transformation. It is not simply given in terms of the Lorentz contraction because the information about the moving surface is delivered by the light rays crossing it, rather than from measurements made by two different but simultaneous observers. If we imagine a surface element $d \sigma^{\prime}$ oriented perpendicular to the plane of $\widehat{\boldsymbol{k}}$ and $\boldsymbol{u}$ with its normal direction at an angle $\theta^{\prime}$ to the relative velocity (hence parallel to a ray in that direction), then the component $d \sigma^{\prime} \sin \theta^{\prime}$ is affected by the change in reference frame. This component becomes equal to $d \sigma \sin \theta$ in the reference frame after tracing the light rays (which define the angle transformation). Hence, by equating these two expressions we have

$$
\frac{d \sigma^{\prime}}{d \sigma}=\frac{\sin \theta}{\sin \theta^{\prime}}=\gamma_{u} \kappa
$$

where we used the standard angle transformation for light rays to get the last expression on the right. In the special case of longitudinal motion, so that $\theta^{\prime}=\theta=0$, this last expression continues to hold (see e.g., Ellis \& Williams 2000).

We are now in possession of the transformation of intensity in the form

$$
I_{\nu}(\nu) \equiv \frac{d^{2} E}{d \Omega d t_{\mathrm{obs}} d \nu d \sigma}=\frac{1}{\gamma_{u}^{2} \kappa^{2}} \frac{d^{2} E^{\prime}}{d \Omega^{\prime} d t^{\prime} d \nu^{\prime} d \sigma^{\prime}} \equiv \frac{I_{\nu^{\prime}}\left(\nu^{\prime}\right)}{\gamma_{u}^{2} \kappa^{2}},
$$

which must be used with $\nu^{\prime}=\nu \gamma_{u} \kappa$. The inverse may be found in the usual way. We see that the spectral index is essentially invariant between the two frames over a small range in angle, which is expected for synchrotron radiation. The comoving radiation energy density is reduced from that measured in the rest frame. The comoving frequency is reduced by the Döppler factor, which might be thought to encourage self-absorption in the comoving frame. However, the electron number density is also reduced by $\gamma_{u}$. If, moreover, the electric field in the source frame is zero, then the perpendicular magnetic field transformation is given by $B_{\perp}^{\prime}=B_{\perp} / \gamma_{u}$. This reduction also offsets the frequency reduction. In the end, the source absorption coefficient is virtually invariant (for $p=2$ ) relative to the rest coefficient.

Another important invariant is the polarization of the source, which we discuss in Section 4.4 and Appendix C. This 
invariance is due to the invariance of the phase of the electromagnetic wave.

In summary, the above relations show that, unless the jet outflow is highly relativistic (i.e., $\gamma_{u} \gg 1$ ), measurements in the observer's rest frame are adequate to describe the instrinsic angles, energy, and magnetic fields of the source. Note that $\gamma_{u} \sim 1$ still encompasses the possibility of very rapid jet speeds. For example, $u=0.5 c \Rightarrow \gamma_{u}=1.15$.

We have neglected the cosmological redshift because NGC 4845 is so close. However, this affects the observed frequency by a well-known conversion from the local rest frame frequency $(\propto 1 /(1+z))$.

\section{APPENDIX C \\ SPECTRAL EVOLUTION OF A SIMPLE ENSEMBLE OF RELATIVISTIC ELECTRONS}

Let us initially assume that the source is, in part, a stationary ensemble of relativistic electrons that is the result of a unique acceleration and that the event that has produced the acceleration occurred at the time of the hard X-ray flare at time, $t_{0}$.

For an individual electron, the synchrotron lifetime, $t_{\mathrm{s}}$, may be written as

$$
t_{\mathrm{s}}=\frac{464}{B_{\perp}(-2)^{3 / 2} \nu_{5}^{1 / 2}} \quad \text { years, }
$$

where $B_{\perp}(-2)$ is the perpendicular (to the electron orbit) magnetic field in units of $10^{-2} \mathrm{G}$ and $\nu_{5}$ is the peak frequency in units of $5 \mathrm{GHz}$. The critical frequency, which is essentially the peak frequency produced by electrons of a given energy, implies that

$$
\gamma_{e}=345 \frac{\nu_{5}^{1 / 2}}{B_{\perp}(-2)^{1 / 2}} .
$$

where $\gamma_{e}$ is the Lorentz factor of the relativistic electron radiating at $5 \mathrm{GHz}$.

For an ensemble of particles in a magnetic field, emission near the spectral turnover will be dominated by those electrons of energy, $\gamma_{e} m_{e} c^{2}$, where $m_{e}$ is the electron mass and $c$ is the speed of light. Thus, from Equation (52), one expects the peak frequency to decline as $\left(t / t_{0}\right)^{-2}$.

From Table 7 , we see that the peak frequencies are in the ratio $\nu_{T_{2}} / \nu_{T_{1}}=4.03 / 4.87=0.83$ after $T_{1}+56$ days, and in the ratio $\nu_{T_{3}} / \nu_{T_{1}}=3.21 / 4.87=0.66$ after $T_{1}+196$ days. Taking $T_{1}$ to be 342 days after the X-ray peak at $t_{0}$ (Section 4$)$, the expected ratios are $\left(\left(T_{1}+56\right) / T_{1}\right)^{-2}=0.74$ and $\left(\left(T_{1}+196\right) / T_{1}\right)^{-2}=0.40$, respectively. The dependence of the peak frequency on time is much closer to being linear than it is to being the quadratic variation expected from such a stationary ensemble emitting near the peak of the spectrum.

For $\nu_{5}=1$ (a spectral turnover of $5 \mathrm{GHz}$; Figure 6), for fields even as large as $0.04 \mathrm{G}$ (a comfortable upper limit; see Artyukh et al. 2008) the lifetime of particles radiating at the spectral turnover is about 58 years and $\gamma_{e}=173$. Higher energy particles by about a factor of 100 would be evolving substantially over our timescale. However, the corresponding frequency is in the infrared. Allowing for the magnetic field to change over the course of the observations is the basis of our explanation of the frequency peak time dependence in the SSA model (see Section 4.3). However, in Equation (52), either the $t^{-1}$ or $t^{-2}$ time dependence in the magnetic field gives quite the wrong time dependence for the peak frequency. Moreover, the evolution timescale becomes longer with a weakening field, which results in an even poorer fit. We observe substantial evolution in less than half a year at $\mathrm{GHz}$ frequencies, however, which tends to exclude this simple picture even if the peak frequency ratios were acceptable.

We conclude that the behavior of a source in which there has been a unique acceleration of electrons and whose emission is now fading with time does not match the observations.

\section{APPENDIX D THERMAL ABSORPTION}

Can thermal absorption explain the low frequency cutoff? The thermal absorption coefficient may be written, by ignoring some small logarithmic terms, as (Pacholczyk 1970)

$$
\kappa_{\mathrm{ec}_{\nu}} \approx \frac{n_{\mathrm{ec}}^{2}}{T_{4}^{3 / 2} \nu(9)^{2}} \times 10^{-25} \mathrm{~cm}^{-1},
$$

where the subscript, ec, refers to "cold" (i.e., non-relativistic, or thermal) electrons; the frequency, $\nu(9)$, is in $\mathrm{GHz}$; and the temperature is in units of $10^{4} \mathrm{~K}$. Multiplying this by the line of sight distance, $s$, and requiring the product to be one (for unity optical depth) shows that, on a scale of $10^{17} \mathrm{~cm}$ in the optically thick limit $(\nu(9) \sim 1)$, we need a cold plasma density of $n_{\mathrm{ec}} \approx 10^{4} \mathrm{~cm}^{-3}$. This might be in a "sheath" around the source (i.e., within or very near the cone). On a scale of one kpc, only $\approx 60 \mathrm{~cm}^{-3}$ is necessary. Such an "H II region" might easily intervene along the line of sight in an edge-on galaxy.

However, if we calculate the effective spectral index, $\alpha_{\text {eff }}$ (i.e., the observed spectral index that results from a synchrotron spectrum, $\left.I_{\nu(o)}=C \nu^{\alpha}\right)$, with an additional absorbing thermal screen, then the observed spectrum will be

$$
I_{\nu}=I_{\nu}(o) e^{-\left(\kappa_{\mathrm{ec}_{\nu}} s\right)}=C \nu^{\alpha} e^{-\left(\kappa_{\mathrm{ec}_{\nu}} s\right)} .
$$

Differentiating this spectrum with respect to frequency and noting the $\nu^{-2}$ dependence of $\kappa_{\mathrm{ec}_{\nu}}$ (Equation (54)) results in an observed spectral index of

$$
\alpha_{\text {eff }}=\alpha+2 \kappa_{\nu} s .
$$

For a non-thermal spectral index of $\alpha=-0.5$ (Table 3), we calculate for $\kappa_{\mathrm{ec}_{\nu}} s=1$ that $\alpha_{\mathrm{eff}}=1.5$, which is too steep (again, Table 3). At $\nu(9) \geqslant 3$, the absorption coefficient is down by a factor of 10. Therefore, we obtain $\alpha_{\text {eff }}=-0.3$, which does not fit the spectra in Figure 6. Thus, thermal absorption does not describe the turnover in the spectrum, and SSA (Section 4.2.1) is preferred. A more detailed analysis based on best fits to the data is given in the text, but our basic conclusion remains.

\section{APPENDIX E \\ CIRCULAR AND LINEAR POLARIZATION IN THE BECKERT AND FALCKE FORMULATION}

A detailed calculation of the circularly polarized flux from the jet model described in Appendix A must be left for elsewhere. This calculation was done in Jones (1988), although frequency dependences for the transition region were not given in that paper. However, a quantitative explanation of how linear polarization is converted to circular polarization is indeed possible, as will be described here. 
In this section we adhere closely to the formulation in the appendices of Beckert \& Falcke (2002). We adopt an isotropic relativistic plasma with a power law energy distribution having $p=2$ ( $s$ in their notation), as suggested by our spectral index in the optically thin limit (Table 3). This plasma state implies that $\kappa_{U}=\eta_{U}=h_{Q}=0$, using the notation of Beckert \& Falcke (2002) for the $U$ absorption coefficient, the $U$ emissivity, and the extraordinary wave conversion coefficient. Our only additional assumptions are to take $\kappa_{V}$ as negligible and to neglect $\kappa_{Q}^{2} V$ compared to $d^{2} V / d s^{2}$. The former assumption is justified by the stonger decline with frequency (by a factor $\left.\sqrt{\nu_{B_{\digamma}} / \nu}\right)$ of $\kappa_{V}$ compared with the remaining absorption coefficients. The latter assumption neglects the second-order change in $V$ by absorption over the whole source, compared to a local second-order change.

The ultrarelativistic conversion coefficient given in Sazonov (1969) requires taking a limit as $p \rightarrow 2$, which we obtain as

$$
\kappa_{\mathrm{c}}=2 \kappa_{o}(\nu)\left(\frac{\nu_{B}}{\nu}\right)(\sin \phi)^{2} \ln \left(\gamma_{o} \sqrt{\frac{\nu_{B}}{\nu}}\right)
$$

where the scaling opacity (proportional to the Faraday absorption coefficient $\kappa_{\mathrm{F}}$ ) is

$$
\kappa_{o} \equiv \frac{\pi \nu_{B}}{c} \frac{\nu_{\mathrm{p}}^{2}}{\nu^{2}} .
$$

The plasma frequency $\nu_{\mathrm{p}}$ is

$$
\nu_{\mathrm{p}}^{2}=\frac{n_{e} e^{2}}{\pi m_{e}},
$$

and $\gamma_{o}$ is the minimum Lorentz factor in the distribution.

A straightforward but tedious manipulation of the equations in Appendix A of Beckert \& Falcke (2002) using these assumptions, yields the following solution for the Stokes parameters as a function of path length in the source, $s$ :

$$
\begin{aligned}
V_{\nu}= & \frac{\eta_{V}}{\kappa_{I} \kappa^{2}}\left(\kappa_{I}^{2}-\kappa_{Q}^{2}+\kappa_{\mathrm{F}}^{2}\right)\left(1-e^{-\kappa_{I} s}\right) \\
& +\frac{\eta_{V} \kappa_{I}}{\kappa^{4}}\left(\kappa_{\mathrm{c}}^{2}-\kappa_{I}^{2}\right) e^{-\kappa_{I} s}(1-\cos \kappa s+(\tan \Phi) \sin \kappa s), \\
U_{\nu}= & \frac{\eta_{V}}{\kappa_{\mathrm{c}} \kappa^{3}}\left(\kappa_{\mathrm{c}}^{2}-\kappa_{I}^{2}\right)\left(\kappa_{I} e^{-\kappa_{I} s}(\sin \kappa s+(\tan \Phi) \cos \kappa s)-\kappa\right), \\
Q_{\nu}= & \frac{\kappa_{\mathrm{c}}}{\kappa_{\mathrm{F}}} V+\frac{\eta_{V} \kappa_{I}}{\kappa_{\mathrm{c}} \kappa_{\mathrm{F}} \kappa^{2}}\left(\kappa_{\mathrm{c}}^{2}-\kappa_{I}^{2}\right)\left(e^{-\kappa_{I} s}(\cos \kappa s\right. \\
& -(\tan \Phi) \sin \kappa s)-1) .
\end{aligned}
$$

The total intensity may be found from

$$
\begin{aligned}
I_{\nu}= & \frac{\eta_{I}}{\kappa_{I}}\left(1-e^{-\kappa_{I} s}\right)-\frac{\kappa_{\mathrm{c}} \kappa_{Q}}{\kappa_{\mathrm{F}}} e^{-\kappa_{I} s} \int^{s} V_{\nu} d s \\
& +\frac{\kappa_{Q}}{\kappa_{\mathrm{F}}}\left(U_{o} e^{-\kappa_{I} s}-U_{\nu}\right)+U_{o} e^{-\kappa_{I} s} .
\end{aligned}
$$

We note that in an optically thick region $\left(\kappa_{I} L\right.$ large and $\kappa_{Q} / \kappa_{\mathrm{F}}$ small), $I_{\nu} \approx \eta_{I} / \kappa_{I}$, which is proportional to $\nu^{5 / 2}$ as expected (in this appendix, $L$ is the total path length through the source, whereas $s$ is a general path length coordinate).

The initial conditions are $V(0)=Q(0)=0, \quad I(0)=U(0)=$ $U_{o}$, and we have set to zero an arbitrary constant of integration that gives a spurious linear increase of $V$ with $s$. The coefficients, $\kappa_{I}, \kappa_{Q}=(9 / 13) \kappa_{I}, \kappa_{\mathrm{F}}$, and the emissivity, $\eta_{V}$, are as in Beckert \& Falcke (2002). In addition, we have defined

$$
\kappa^{2}=\left(\kappa_{\mathrm{c}}^{2}-\kappa_{Q}^{2}+\kappa_{\mathrm{F}}^{2}\right),
$$

and

$$
\tan \Phi=\frac{\kappa}{\kappa_{I}}+\frac{\kappa_{\mathrm{c}} \kappa^{3}}{\kappa_{I}\left(\kappa_{\mathrm{c}}^{2}-\kappa_{I}^{2}\right)} \frac{U_{o}}{\eta_{V}} .
$$

The linear dependence on $\eta_{V}$ is a result of isotropy, our boundary conditions, and the identity $\kappa_{I} \eta_{Q}-\kappa_{Q} \eta_{I}=0$, which hold for the coefficients (Beckert \& Falcke 2002). However the solution in detail as displayed in the following shows that $V$ is mainly dependent on $U_{o}$, as is expected.

We turn to an analysis of the frequency dependences of these solutions. It is convenient to express all frequencies in terms of one $\mathrm{GHz}$ (denoted $\nu_{9}$ ), and to let all emission and absorption coefficients have their frequency independent values at one $\mathrm{GHz}$ when expressed with their usual names () modified to $\tilde{()}$. Thus $\eta_{V}=\tilde{\eta}_{V} / \nu_{9}$ and $\kappa_{\mathrm{c}}=\tilde{\kappa}_{\mathrm{c}} / \nu_{9}^{3}, \kappa_{I}=\tilde{\kappa}_{I} / \nu_{9}^{3}$ and so on. Only $\kappa$ requires a slight complication because $\kappa_{\mathrm{F}}=\tilde{\kappa}_{\mathrm{F}} / \nu_{9}^{2}$. Hence, from the definition, $\kappa$ may either have the form $\kappa=\tilde{\kappa} / \nu_{9}^{3}$ if $\kappa_{\mathrm{c}}$ and $\kappa_{Q}$ are dominant; alternately, $\kappa=\tilde{\kappa}_{\mathrm{F}} / \nu_{9}^{2}$ if $\kappa_{\mathrm{F}}$ is dominant. Other than in these extreme cases, $\kappa$ presents a mixed frequency behavior.

In the next two subsections, we proceed by assuming that $\kappa_{I} L \ll 1$ ( $L$ is the path length through the source) and consider the limits of small and large Faraday depths.

\section{E.1 The Case of Small Faraday Depth}

In the first case, let us assume that $\kappa_{\mathrm{F}}$ is small compared with $\kappa_{\mathrm{c}}$ or $\kappa_{Q}$. We note that this requires the minimum Lorentz factor $\gamma_{o}$ to be essentially equal to the $\gamma_{e}$ associated with the peak frequency in the radio band. Then the solution for $V$ becomes, to first order in $\kappa_{I} L$ and $\kappa L$,

$$
V \approx \eta_{V} L \frac{\kappa_{I}\left(\kappa_{\mathrm{c}}^{2}-\kappa_{I}^{2}\right)}{\kappa^{3}}(\tan \Phi)+\eta_{V} L\left(1+\frac{\kappa_{I}^{2}-\kappa_{\mathrm{c}}^{2}}{\kappa^{2}}\right) .
$$

In addition, with the frequency dependences displayed and taking $\kappa_{\mathrm{F}}$ small, the expression for the circularly polarized intensity (cpi) becomes

$$
V \approx \tilde{\eta}_{V} L \frac{\tilde{\kappa}_{I}\left(\tilde{\kappa}_{\mathrm{c}}^{2}-\tilde{\kappa}_{I}^{2}\right)}{\left(\tilde{\kappa}_{\mathrm{c}}^{2}-\tilde{\kappa}_{Q}^{2}\right)^{3 / 2}} \frac{\tan \Phi}{\nu_{9}}+\tilde{\eta}_{V} L \frac{\tilde{\kappa}_{I}^{2}-\tilde{\kappa}_{Q}^{2}}{\tilde{\kappa}_{\mathrm{c}}^{2}-\tilde{\kappa}_{Q}^{2}} \frac{1}{\nu_{9}},
$$

where

$$
\tan \Phi=\frac{\sqrt{\tilde{\kappa}_{\mathrm{c}}^{2}-\tilde{\kappa}_{Q}^{2}}}{\tilde{\kappa}_{I}}+\frac{U_{o}}{\tilde{\eta}_{V}} \frac{\tilde{\kappa}_{\mathrm{c}}}{\tilde{\kappa}_{I}} \frac{\left(\tilde{\kappa}_{\mathrm{c}}^{2}-\tilde{\kappa}_{Q}^{2}\right)^{3 / 2}}{\tilde{\kappa}_{\mathrm{c}}^{2}-\tilde{\kappa}_{I}^{2}} \frac{1}{\nu_{9}^{2}} .
$$

Consequently, after inserting Equation (66) into Equation (65) we have simply

$$
V \approx \frac{\tilde{\eta}_{V} L}{\nu_{9}}+\frac{U_{o} \tilde{\kappa}_{\mathrm{c}} L}{\nu_{9}^{3}}
$$

Thus when the Faraday absorption is small compared to relativistic conversion, there is an internal generation term in the circular polarized flux $\propto \nu^{-1}$, plus a conversion term $\propto \nu^{-3}$. It is the latter term that we are particularly interested in because it dicates the linear to circular polarization, and also has the steep spectral behavior that is observed (Table 3). However, the 
first term could also contribute because the spectral indices, on average, tend to be slightly flatter than -3 .

A numerical calculation using the values given in Beckert \& Falcke (2002) and our formula for $\kappa_{\mathrm{c}}$ gives (where $L(17)$ is in units of $10^{17} \mathrm{~cm}$ and $\gamma_{o}=200$ )

$$
\begin{aligned}
\tilde{\kappa}_{\mathrm{c}} L \approx & 7.7 \times 10^{-3} L(17) B^{2}(-2) n_{e}(100), \\
\tilde{\eta}_{V} L \approx & 0.28 B^{2}(-2) n_{e}(100) L(17) \\
& \times 10^{8}\left\{=22 \theta_{\mathrm{s}}^{2} B^{2}(-2) n_{e}(100) L(17) \mathrm{mJy}\right\} .
\end{aligned}
$$

The actual circularly polarized flux measured at the antenna $V_{\nu A}=\Omega_{\mathrm{s}} V$. Thus the first term in Equation (67) must be multiplied by the factor $\Omega_{\mathrm{s}}$. This factor is $\approx 7.8 \times 10^{-7} \theta_{\mathrm{s}}^{2}$, so the numerical factor in the second of Equations (68) becomes $22 \theta_{\mathrm{s}}^{2}$. The source angular size is expressed in milli-arcsec, and may be as small as 0.1 when $L(17)=1$. For the second term in Equation (67), we consider $U_{o}$ as if it has already been corrected to flux density, thus Equations (67) and (68) together give

$$
\begin{aligned}
V \approx & \frac{22 \theta_{\mathrm{s}}^{2} B^{2}(-2) n_{e}(100) L(17)}{\nu_{9}} \\
& +\frac{7.7 \times 10^{-3} U_{o} L(17) B^{2}(-2) n_{e}(100)}{\nu_{9}^{3}} \mathrm{mJy}
\end{aligned}
$$

Hence the first term in $V$ may contribute to our circular polarization if $B(-2) \approx 4$ and the relativistic electron density $n_{e}$ is as large as $100 \mathrm{~cm}^{-3}$ at $1.5 \mathrm{GHz}(V=2.3 \mathrm{mJy})$. Under these conditions, the second term would require an $\operatorname{lpf} U_{o} \approx 60$ $\mathrm{mJy}$ in order to produce the observed circularly polarized flux by conversion $(V=2.2 \mathrm{mJy}$; i.e., comparable values that are roughly consistent with our total circularly polarized flux density). However, the second term is favored by our measured in-band values of $\overline{\alpha_{V}}$. The required incident $U_{o}$ that makes the second term comparable to the first term is larger than any lpf (close to 24\%) that we or others observe. We must then consider the exit values of $U$ and $Q$ further, especially after passing through a possible depolarizing screen (cf. O'Sullivan et al. 2013).

The dominant term in the total intensity is approximately the first term in Equation (61). Assuming that both terms in Equation (67) are comparable, we obtain a rough estimate of the percentage cpf as

$$
\frac{\tilde{\eta}_{V}}{\tilde{\eta}_{I}\left(\nu_{9}\right)^{1 / 2}} \approx-\cot \phi \sqrt{\frac{B_{\perp}(-2)}{\nu_{9}}} \times 0.9 \times 10^{-2} .
$$

This can be of order $2 \%$ for $B(-2) \geqslant 4$.

We note that any balanced mixture of the two terms in Equation (67) will change quickly with increasing frequency toward a $\nu^{-1}$ behavior. Moreover the cpi cannot be much larger than $\tilde{\kappa}_{\mathrm{c}} L U_{o}$ (cf. O'Sullivan et al. 2013). Because of the harmonic functions of $\kappa s$ in the general expression for $V$, this cpi state also changes rapidly in the optical depth transition zone as we require.

Unfortunately, proceeding in the same fashion as for the circular polarization, we find that the Stokes $U$ is modified only by absorption. It becomes

$$
U=U_{o}\left(1-\frac{\tilde{\kappa}_{I} L}{\nu_{9}^{3}}\right)
$$

and so is essentially unchanged from $U_{o}$. The latter quantity is thus to be constrained by the small value of lpf that we observe. One must appeal to a depolarizing screen to remove the linear polarization (e.g., O'Sullivan et al. 2013) if it is really of this order. We note that a magnetic field as large as $B(-2)=10$ (e.g., Artyukh et al. 2008) would reduce the required $U_{o}$ to a more reasonable $3 \%-4 \%$, but this would still have to be removed by an inhomogeneous, depolarizing screen to satisfy our limit.

Finally, we calculate Stokes $Q$ in the same approximation and limit. When there is very little Faraday rotation we find that $Q \approx 0$ in lowest order, provided that $\tilde{\kappa} \approx \tilde{\kappa}_{\mathrm{c}}$, and that this quantity is much larger than either $\tilde{\kappa}_{I}$ or $\tilde{\kappa}_{Q}$.

\section{E.2 The Case of Large Faraday Depth}

A second limiting case arises when the Faraday depth is large so that $\kappa_{\mathrm{F}}$ is large compared with $\kappa_{\mathrm{c}}$ or $\kappa_{Q}$ (and therefore $\kappa_{I}$, since for $p=2$ this coefficient is larger only by the factor 13/9). In this limit $\kappa \approx \tilde{\kappa}_{\mathrm{F}} / \nu_{9}^{2}$ and we find at small $\kappa_{I} L$ that

$$
V \approx \frac{\tilde{\eta}_{V} L}{\nu_{9}}+\tilde{\eta}_{V} \frac{\tilde{\kappa}_{I}\left(\tilde{\kappa}_{\mathrm{c}}^{2}-\tilde{\kappa}_{I}^{2}\right)}{\tilde{\kappa}_{\mathrm{F}}^{4}} \frac{\tan \Phi}{\nu_{9}^{4}} \sin \left(\kappa_{\mathrm{F}} L\right) .
$$

Moreover

$$
\tan \Phi=\frac{\tilde{\kappa}_{\mathrm{F}}}{\tilde{\kappa}_{I}} \nu_{9}+\frac{\tilde{\kappa}_{\mathrm{c}}}{\tilde{\kappa}_{I}} \frac{\tilde{\kappa}_{\mathrm{F}}^{3}}{\tilde{\kappa}_{\mathrm{c}}^{2}-\tilde{\kappa}_{I}^{2}} \frac{U_{o}}{\tilde{\eta}_{V}} \nu_{9},
$$

and hence

$$
V \approx \frac{\tilde{\eta}_{V}}{\nu_{9}^{3}} \frac{\tilde{\kappa}_{\mathrm{c}}^{2}-\tilde{\kappa}_{I}^{2}}{\tilde{\kappa}_{\mathrm{F}}^{3}} \sin \left(\kappa_{\mathrm{F}} L\right)+\frac{\tilde{\eta}_{V} L}{\nu_{9}}+\frac{U_{o} \tilde{\kappa}_{\mathrm{c}}}{\tilde{\kappa}_{\mathrm{F}} \nu_{9}^{3}} \sin \left(\kappa_{\mathrm{F}} L\right)
$$

The terms in $\tilde{\eta}_{V}$ must be assumed to be reduced numerically by $\Omega_{\mathrm{s}}$ to give the observed flux, as in the previous case.

The first term in Equation (74) is small compared with the second term around $\nu_{9}=1$, according to our limiting assumption. Thus, the combined frequency dependence due to the second and third terms is not changed from the small Faraday rotation limit. The magnitude of the conversion term (proportional to $U_{o}$ ) is however reduced by the factor $\tilde{\kappa}_{\mathrm{c}} / \tilde{\kappa}_{\mathrm{F}}$, which is assumed to be quite small. In fact, this ratio is (see formulae in Beckert \& Falcke 2002)

$$
\frac{\tilde{\kappa}_{\mathrm{c}}}{\tilde{\kappa}_{\mathrm{F}}} \approx 0.75 \times \frac{\nu_{B} \gamma_{o}^{3}}{\nu} \quad \frac{\sin ^{2} \phi}{\cos \phi}\left(1+\frac{\ln \left(\nu_{B} / \nu\right)}{2 \ln \gamma_{o}}\right) .
$$

This is very small unless $\gamma_{o}$ approaches 100 or more, which becomes the previous case. Hence in this case the conversion term is not important compared to the second synchrotron emission term (cf. Equation (68) for $\tilde{\eta}_{V}$ ). This can give sufficient cpf as we have seen, and it is not oscillatory. However, the frequency dependence is $\nu^{-1}$, rather than the behavior we observe.

For completeness, and in order to add insight into the effects of Faraday inhomogeneity, we calculate the Stokes parameters $Q$ and $U$ in the Faraday dominant limit as

$$
U \approx U_{o} \cos \left(\kappa_{\mathrm{F}} L\right)
$$

and

$$
Q=-U_{o} \sin \left(\kappa_{\mathrm{F}} L\right) .
$$


The only way to suppress a large $U_{o}$ is to imagine many cells with random properties. Then $L$ is replaced by a characteristic $s$ for each cell and, in the mean, the linear polarization is suppressed (Beckert \& Falcke 2002). The same suppression would occur in a depolarizing sheath.

\section{APPENDIX F \\ X-RAY EMISSION VIA INVERSE COMPTON}

Appendix A outlines the jet model in which we imagine that the X-ray emission around the time of the peak of the X-ray light curve comes from a small X-ray "lobe" (see Figure 7). We wish to link the currently observed radio emission to a time approximately a year earlier to determine whether conditions at that time could account for the X-ray peak flux via inverse Compton upscattering, also known as Synchrotron SelfCompton, or SSC. As before, our calculations are order of magnitude to check for feasibility.

As indicated in the text (Equation (53)), the critical frequency, $\nu_{\mathrm{c}}(\mathrm{Hz})$, for a relativistic electron of Lorentz factor, $\gamma_{e}$, in a magnetic field of strength, $B$, is

$$
\nu_{\mathrm{c}}=4.2 \times 10^{4} \gamma_{e}^{2} B_{\perp}(-2)
$$

where $B_{\perp}(-2)$ is the perpendicular magnetic field strength ( $B_{\perp}=B \sin \theta$, with $\theta$ the pitch angle). Hence, for an electron to radiate in the radio regime at $5 \mathrm{GHz}$ (the peak of the spectrum at time $T_{1}$; Table 7), in a magnetic field of $B_{\perp}(-2)=1 \mathrm{a}$ Lorentz factor of $\gamma_{e} \approx 347$ is required. Such a value could describe conditions in the radio source around the time of the radio observations.

We need to consider the earlier time in the X-ray lobe at which $\gamma_{e}$ and $B_{\perp}(-2)$ are both higher and the source size is smaller. The SSC requirement is $\nu_{\mathrm{X}}=\gamma_{e}^{2} \nu_{\mathrm{c}}$, so from the above equation,

$$
\nu_{\mathrm{X}}=4.2 \times 10^{4} \gamma_{e}^{4} B_{\perp}(-2)
$$

The hard X-ray light curve of Nikolajuk \& Walter (2013) applies to the energy range, $17.3-80 \mathrm{keV}$, and we adopt $30 \mathrm{keV}$, or $\nu_{\mathrm{X}}=7.2 \times 10^{18} \mathrm{~Hz}$, as the $\mathrm{X}$-ray frequency. Also, for a jet of constant velocity, $B_{r} \propto r^{-2}$ (see Appendix A), so for an X-ray lobe that is a factor of 10 smaller than the current radio source size $\left(10^{16}\right.$ rather than $10^{17} \mathrm{~cm}$, or 0.1 mas rather than 1 mas), the magnetic field strength can be estimated to be $B_{\perp}(-2)=100$ at the earlier time. We now find the Lorentz factor for relativistic electrons in the X-ray lobe, which from Equation (79) is $\gamma_{e}=1109$. Then, by Equation (78), the synchrotron critical frequency would be $\nu_{\mathrm{c}}=5.5 \times 10^{12} \mathrm{~Hz}$ or $\lambda_{\mathrm{c}}=55 \mu \mathrm{m}$. Thus, the synchrotron emission in the $\mathrm{X}$-ray lobe would be in the infrared at the earlier time. Although not required for this development, one can show via standard formulae, that such emission would be optically thin.

We now need to calculate the infrared flux in the X-ray lobe, given these conditions. First, we find the specific intensity of the synchrotron emission, specifying infrared with the subscript, IR,

$$
\begin{aligned}
I_{\nu_{\mathrm{IR}}}= & \epsilon_{\nu_{\mathrm{IR}}} s_{\mathrm{X}} \\
= & C_{5}(p) N_{0_{\mathrm{IR}}} B_{\mathrm{IR}}(p+1) / 2\left(\nu_{\mathrm{IR}} / 2 C_{1}\right)^{(1-p) / 2} \\
& \times\left(\sin \left(\theta_{\mathrm{IR}}\right)\right)^{(1+p) / 2} s_{\mathrm{X}}
\end{aligned}
$$

where $\epsilon_{\nu \mathrm{R}}$ is the emissivity and $s_{\mathrm{X}}$ is the line of sight distance through the X-ray lobe. Here, $C_{5}(p)$ and $C_{1}$ are Pacholczyk's constants (Pacholczyk 1970) for the electron energy spectral index, $p$, and $N_{0_{\mathrm{IR}}}$ is a normalizing factor for the energy spectrum (cf. Section 4.3). We have explicitly expressed the magnetic field, $B_{\mathrm{IR}}$, separated from $\sin \theta_{\mathrm{IR}}$, because in the next step we will compare current radio values to IR values at the earlier time and, when $\gamma_{e}$ changes, so does the pitch angle.

We now multiply and divide Equation (80) by the same expression for the (current) radio emission, $I \nu_{\mathrm{R}}$, where R refers to the radio, finding after some manipulation,

$$
\begin{aligned}
I_{\nu_{\mathrm{IR}}}= & I \nu_{\mathrm{R}}\left(\frac{N_{0 \mathrm{IR}}}{N_{0 \mathrm{R}}}\right)\left(\frac{B_{\mathrm{IR}}}{B_{\mathrm{R}}}\right)^{(1+p) / 2} \\
& \times\left(\frac{\sin \left(\theta_{\mathrm{IR}}\right)}{\sin \left(\theta_{\mathrm{R}}\right)}\right)^{(1+p) / 2}\left(\frac{\nu_{\mathrm{IR}}}{\nu_{\mathrm{R}}}\right)^{(1-p) / 2}\left(\frac{s_{\mathrm{X}}}{s_{\mathrm{R}}}\right) .
\end{aligned}
$$

A conversion to flux, $S_{\mathrm{IR}}$, requires multiplying by the source solid angle and by the frequency. If we take $p=2$, which is implied from the frequency spectral index in the radio, then Equation (81) becomes

$$
\begin{aligned}
S_{\mathrm{IR}}= & S_{\mathrm{R}}\left(\frac{N_{0 \mathrm{IR}}}{N_{0 \mathrm{R}}}\right)\left(\frac{B_{\mathrm{IR}}}{B_{\mathrm{R}}}\right)^{3 / 2}\left(\frac{\sin \left(\theta_{\mathrm{IR}}\right)}{\sin \left(\theta_{\mathrm{R}}\right)}\right)^{3 / 2} \\
& \times\left(\frac{\nu_{\mathrm{IR}}}{\nu_{\mathrm{R}}}\right)^{-1 / 2}\left(\frac{s_{\mathrm{X}}}{s_{\mathrm{R}}}\right) .
\end{aligned}
$$

We can now relate the radio quantities, which are mostly known, to the infrared quantities at the earlier time to estimate $S_{\mathrm{IR}}$. We take $S_{\mathrm{R}}=\nu_{\mathrm{R}} S_{\nu_{\mathrm{R}}}$, where $\nu_{\mathrm{R}}=5 \times 10^{9} \mathrm{~Hz}$ and $S_{\nu_{\mathrm{R}}}=433 \mathrm{mJy}$ (Table 7 for time $T_{1}$ ) to find $S_{\mathrm{R}}=2.1 \times 10^{-14}$ $\mathrm{erg} \mathrm{s}^{-1} \mathrm{~cm}^{-2}$. For an adiabatically expanding source, we found (Section 4.3), that $N_{0 \mathrm{IR}} / N_{0 \mathrm{R}} \propto\left(t_{\mathrm{IR}} / t_{\mathrm{R}}\right)^{-2 / 3}$, where $t_{\mathrm{R}}$ and $t_{\mathrm{IR}}$ are the times for the radio emission and IR emission, respectively. We can take $t_{\mathrm{IR}} / t_{\mathrm{R}} \approx 1 / 2$, because the time from the outburst to the radio observations is about twice the time from the outburst to the X-ray peak, leading to $N_{0 \mathrm{IR}} / N_{0 \mathrm{R}} \approx 1.6$. The ratio, $B_{\mathrm{IR}} / B_{\mathrm{R}}=100$, from above. For the pitch angle, we take $\sin \left(\theta_{\mathrm{IR}}\right) / \sin \left(\theta_{\mathrm{R}}\right)=\gamma_{e \mathrm{R}} / \gamma_{\text {eIR }}=$ $347 / 1109=0.31$. The ratio of frequencies is $\nu_{\mathrm{IR}} / \nu_{\mathrm{R}}=$ $\left(5.5 \times 10^{12}\right) /\left(5 \times 10^{9}\right)=1.1 \times 10^{3}$ and the ratio of sizes is $s_{\mathrm{X}} / s_{\mathrm{R}}=0.1$.

The result is $S_{\mathrm{IR}}=1.9 \times 10^{-11} \mathrm{erg} \mathrm{s}^{-1} \mathrm{~cm}^{-2}$.

It remains to compute the X-ray flux from SSC. From Equations (23) and (24) expressed in a more convenient form in terms of the infrared synchrotron emission in the shocked region that emits the $\mathrm{X}$-rays,

$$
S_{\mathrm{X}_{\mathrm{SSC}}}=S_{\mathrm{IR}} \frac{U_{\mathrm{rad}}}{U_{\mathrm{mag}}}=5.7 \times 10^{12} \frac{S_{\mathrm{IR}}^{2}}{\theta_{\mathrm{s}}^{2} B_{\mathrm{IR}}(-2)^{2}}
$$

for $S_{\mathrm{X}_{\mathrm{SSC}}}, \quad S_{\mathrm{IR}}$ in erg s${ }^{-1} \mathrm{~cm}^{-2}, \theta_{s}$ in mas, and $B_{\mathrm{IR}}(-2)$ in units of $10^{-2} \mathrm{G}$. With the value of $S_{\mathrm{IR}}, \theta_{\mathrm{S}}=0.1$ and $B_{\mathrm{IR}}(-2)=100$ (see previous), we find, $U_{\text {rad IR }} / U_{\mathrm{mag}}=1.1$ and $S_{\mathrm{XSSC}_{\mathrm{SSC}}}=2.0 \times$ $10^{-11} \mathrm{erg} \mathrm{s}^{-1} \mathrm{~cm}^{-2}$. The peak of the $\mathrm{X}$-ray light curve is $S_{\mathrm{X}} \approx 8.0 \times 10^{-11} \mathrm{erg} \mathrm{s}^{-1} \mathrm{~cm}^{-2}$.

Thus, our estimated X-ray flux from SSC falls short of the observed X-ray peak by only a factor of four. The result is quite sensitive to the choice of magnetic field in the radio, which was taken as $B_{\mathrm{R}}(-2)=1$ previously. For example, if 
$B_{\mathrm{R}}(-2)=4$ then $U_{\mathrm{rad}_{\mathrm{IR}}} / U_{\mathrm{mag}}=0.06$ and $S_{\mathrm{X}_{\mathrm{SSC}}}=1.0 \times 10^{-12}$ $\mathrm{erg} \mathrm{s}^{-1} \mathrm{~cm}^{-2}$, which falls short of explaining the X-ray peak. If $B_{\mathrm{R}}(-2)=0.5$, then $S_{\mathrm{X}_{\mathrm{SSC}}}=1.0 \times 10^{-10} \mathrm{erg} \mathrm{s}^{-1} \mathrm{~cm}^{-2}$, which is more than adequate to explain the X-ray peak; however, $U_{\mathrm{rad}} / U_{\mathrm{mag}}=4.6$ is uncomfortably large.

Because the result is so sensitive to the magnetic field, one needs to examine it more closely. We have assumed that $B_{r} \propto r^{-2}$, which only accounts for geometric expansion. Yet, our jet model indicates that the $\mathrm{X}$-ray lobe is a shocked region that should enhance the magnetic field. If we allow for geometric expansion, as before, but include a modest enhancement to the magnetic field in the X-ray lobe of a factor of 1.8 (a strong shock would give factor of 4) then $U_{\mathrm{rad}_{\mathrm{IR}}} / U_{\mathrm{mag}}=1.1$ and $S_{\mathrm{X}_{\mathrm{SSC}}}=8 \times 10^{-11} \mathrm{erg} \mathrm{s}^{-1} \mathrm{~cm}^{-2}$. With these conditions, SSC can account for the X-ray flux at the peak of the light curve.

\section{REFERENCES}

Artyukh, V. S., Chernikov, P. A., \& Tyul'bashev, S. A. 2008, A\&A, 486, 735 Blandford, R., \& Königl, A. 1979, ApJ, 232, 34

Becker, R. H., Helfand, D. J., White, R. L., et al. 2012, yCat, 8090, 0

Becker, R. H., White, R. L., \& Helfand, D. J. 1995, ApJ, 450, 559

Beckert, T., \& Falcke, H. 2002, A\&A, 388, 1106

Briggs, D. S. 1995, PhD thesis, The New Mexico Institute of Mining and Technology

Brisken, W. 2003, EVLA Memo \#58, "Using GRASP8 to Study the VLA Beam"

Cayatte, V., van Gorkom, J. H., Balkowski, C., \& Kotanyi, C. 1990, AJ, 100,604

Condon, J. J., Cotton, W. D., Greisen, E. W., et al. 1998, AJ, 115, 1693

Cornwell, T. J. 2008, ISTSP, 2, 793

Cornwell, T. J., Golap, K., \& Bhatnagar, S. 2008, ISTSP, 2, 647

Schwab, F. R. 1984, AJ, 89, 1076

Dressel, L. L., \& Condon, J. J. 1978, ApJS, 36, 53

Ellis, G. F. R., \& Williams, R. M. 2000, Flat and Curved Space-Times (2nd ed.; Oxford: Oxford Univ. Press)

Fanti, C. 2009, AN, 330, 120

Filho, M. E., Barthel, P. D., \& Ho, L. C. 2000, ApJS, 129, 93

Gregory, P. C. G., Scott, W. K., Douglas, K., \& Condon, J. J. 1996, ApJS, 103,427

Harnett, J. I. 1982, AuJPh, 35, 321

Henriksen, R. N. 2011, Practical Relativity (Chichester, UK: Wiley)

Henriksen, R. N., Mirabel, F., \& Ptuskin, V. S. 1991, A\&A, 248, 221

Ho, L. C., Filippenko, A. V., \& Sargent, W. L. W. 1995, ApJS, 98, 477
Homan, D. C., \& Wardle, J. F. C. 1999, AJ, 118, 1942

Irwin, J. A., Beck, R., Benjamin, R. A., et al. 2012a, AJ, 144, 43

Irwin, J. A., Beck, R., Benjamin, R. A., et al. 2012b, AJ, 144, 44

Irwin, J. A., Krause, M., English, J., et al. 2013, AJ, 146, 164

Jones, T. W. 1988, ApJ, 332, 678

Karachentsev, I. D., \& Nasonova, O. G. 2013, MNRAS, 429, 2677

Kennett, M., \& Melrose, D. 1998, PASA, 19, 43

Kenney, J. D. P., \& Koopmann, R. A. 1999, AJ, 117, 181

Kenney, J. D. P., van Gorkom, J. H., \& Vollmet, B. 2004, AJ, 127, 3361

Komugi, S., Sofue, Y., Kohno, K., et al. 2008, ApJS, 178, 225

LaMassa, S. M., Cales, S., Moran, E. C., et al. 2015, ApJ, 800, 144

Longair, M. S. 1994, High Energy Astrophysics, Vol. 2 (2nd ed.; Cambridge: Cambridge Univ. Press)

Macquart, J.-P. 2002, PASA, 19, 43

Malkov, M. A., \& Drury, L. O'C. 2001, RPPh, 64, 429

McMullin, J. P., Waters, B., Schiebel, D., Young, W., \& Golap, K. 2007, ASP Conf. Ser. 376, Astronomical Data Analysis Software and Systems XVI, ed. R. A. Shaw, F. Hill \& D. J. Bell (San Francisco, CA: ASP), 127

Melrose, D. B. 1980, Plasma Astrophysics (Vol. 2; London: Gordon and Breach)

Mingaliev, M. G., Sotnikova, Y. V., Mufakharov, T. V., et al. 2013, AstBu, 68, 262

Murphy, E. J., Kenney, D. J. P., Helou, G., et al. 2009, ApJ, 694, 1435

Nikolajuk, M., \& Walter, R. 2013, A\&A, 552, A75

O’Dea, C. P. 1998, PASP, 110, 493

O’Dea, C. P., Baum, S. A., \& Stanghellini, C. 1991, ApJ, 380, 660

Orienti, M., Murgia, M., \& Dallacasa, D. 2010, MNRAS, 402, 1892

Pacholczyk, A. G. 1970, Radio Astrophysics (San francisco: Freeman)

Pacholczyk, A. G. 1973, MNRAS, 163, 29p

Perley, R. A., \& Butler, B. J. 2013, ApJS, 204, 19

Rau, U., \& Cornwell, T. J. 2011, A\&A, 532, A71

Sazonov, V. N. 1969, SvA, 13, 396

Simmons, J. F. L., \& Stewart, B. G. 1985, A\&A, 142, 100

Solanes, J. M., Sanchis, T., Eduard Salvador-Solé, E., et al. 2002, AJ, 124,2440

Springob, C. M., Haynes, M. P., Giovanelli, R., \& Kent, B. R. 2005, ApJS, 160,149

O'Sullivan, S. P., McClure-Griffiths, N. M., Feain, I. J., et al. 2013, MNRAS, 435, 311

Tornikoski, M., Jussila, I., Johansson, P., Lainela, M., \& Valtoaja, E. 2001, AJ, 121, 1306

Tully, R. B. 1982, ApJ, 257, 389

Vaillancourt, J. E. 2006, PASP, 118, 1340

Véron-Cetty, M.-P., \& Véron, P. 2010, A\&A, 518, 10

Vollmer, B., Soida, M., Beck, R., et al. 2013, A\&A, 553, A116

Wang, J.-Z., et al. 2014, ApJ, 788, 32

Wiegert, T., Irwin, J., Miskolczi, A., et al. 2015, AJ, in press

Zauderer, B. A., Berger, E., Soderberg, A. M., et al. 2011, Natur, 476, 425

Zheleznyakov, V. V., \& Koryagin, S. A. 2002, AstL, 28, 727 